\title{
Least Squares Stationary Optimal Control and the Algebraic Riccati Equation
}

\author{
JAN C. WILLEMS, MEMBER, IEEE
}

\begin{abstract}
The optimal control of linear systems with respect to quadratic performance criteria over an infinite time interval is treated. Both the case in which the terminal state is free and that in which the terminal state is constrained to be zero are treated. The integrand of the performance criterion is allowed to be fully quadratic in the control and the state without necessarily satisfying the definiteness conditions which are usually assumed in the standard regulator problem. Frequency-domain and time-domain conditions for the existence of solutions are derived. The algebraic Riccati equation is then examined, and a complete classification of all its solutions is presented. It is finally shown how the optimal control problems introduced in the beginning of the paper may be solved analytically ria the algebraic Riccati equation.
\end{abstract}

\section{INTRODUCTION}

$\mathbf{P}$ ROBABLY the most important result in modern systems theory, both in terms of potential practical and theoretical applications, is the solution of the infinite time least squares problem for stationary linear dynamical systems. It indeed gives one a systematic procedure for computing constant feedback control gains for multipleinput systems based on a performance criterion which admits a simple (albeit not always physically easily motivated) interpretation in terms of the control effort and the error.

It seems therefore worthwhile to study this problem in depth. Although the basic regulator problem is very well understood and widely applied, there are many facets of the quadratic performance criterion problem which remain difficult and vague. Among these are the case in which the integrand in the performance criterion need not be positive or when this integrand is linear in the control (singular problems). Nevertheless, these problems bave important applications, for example, to control problems with conflicting objectives, to problems in network synthesis, in stability theory, second variations, etc.

There are two main areas in control theory where infinite time least squares minimization problems have been developed. On the one hand there is the standard regulator problem of optimal control theory, and on the other hand there are the Lyapunov functions which lead,

Manuscript received July 19, 1971. Paper recommended by S. K. Mitter, Associate Guest Editor. This work was supported by the National Science Foundation under Grant GK 2645 and by the United Kingdom Science Research Council.

The author was with the Department of Applied Mathematics and Theoretical Physics, University of Cambridge, Cambridge, England, on leave from the Department of Electrical Engineering, Massachusetts Institute of Technology, Cambridge, Mass. 02139. He is now with the Electronic Systems Laboratory, M.I.T., Cambridge, Mass. 02139 . via the so-called Kalman-Yacubovich-Popov lemma, to the circle criterion and the Popov criterion in stability theory for feedback systems. Whereas the stability criteria just mentioned appear to be very well known, it is fair to state that their variational interpretation is not appreciated and entirely ignored in most of the recent texts on optimal control theory. This is particularly disappointing since the optimal control problems which lead to these stability criteria are much more intricate and challenging than the standard regulator problem.

This paper started as an effort to unify the existing results. In the course of doing this, it became apparent that some important extensions to the present theory could be successfully pursued. Among the new results presented here is a general treatment of the boundedness of the infima both in the case that the final state is free and that the final state is zero, a necessary and sufficient condition for the existence of real symmetric solutions to the algebraic Riccati equation, and a classification, in terms of certain projection operators, of all the solutions to the algebraic Riccati equation.

Although the least squares optimization problem with linear differential constraints has roots going back to the very beginnings of calculus of variations, its revival and introduction in control theory may safely be credited to Kalman [1]. We should also mention Newton et al. [2], who put forward least squares techniques as a systematic basis for the design of stationary feedback control systems. Many of the results of this paper are inspired by some of the results obtained by Brockett. His work has appeared in various places in the literature and may be found in summarized form in the recent text [3]. Other papers which make ample contact with the results presented here are some of the later papers by Kalman $[4],[5]$, the important paper by Popov [6], and the work of Anderson (see, for example, Anderson [7] and Anderson and Moore [8]).

To those who doubt that such an intensive study of specialized optimization problems as the one studied here is worthwhile, we offer the opinion that these problems remain challenging and have an essentially unlimited range of applications. One will recognize applications in network theory, optimal control, stability theory, detection theory, filtering and prediction theory, identification, numerical analysis, differential games, to name but a few! The theoretically inclined reader will realize that these questions essentially consist of studying a system with an associated performance: these are fundamental system- 
theoretic problems and the subtleties involved in such a study should at least match the subtleties discovered recently in the structure of multivariable systems.

We will only be concerned with linear stationary finitedimensional systems and (consequently) infinite time least squares optimization problems. One may suspect that other than the linearity, none of these assumptions is essential. In fact some of the results may be generalized, mutatis mutandis, to time-varying and distributed parameter systems. We will postulate controllability and observability wherever convenient. We assume all quantities to be real. (This policy will on occasion have to be abandoned when considering eigenvalues, eigenvectors, and transfer function matrices.)

The notation followed is that decreed by the Editor of this issue. Some special notation which occurs is $\lambda(M)$ for an $(n \times n)$ matrix $\boldsymbol{M}$. This denotes an arbitrary eigenvalue of $M$. We will sometimes use the notation $\left\langle x_{1}, x_{2}\right\rangle$, for $x_{1}{ }^{\prime} x_{2}$, and $\|x\|_{P}{ }^{2}$, for $x^{\prime} P x$. The time dependence of functions appearing in integrals is usually deleted. The overbar notation $(-)$ denotes complex conjugation. Finally, we will have the occasion to use the function spaces $L_{2 e}{ }^{+}$and $L_{2 e}{ }^{-}$which are defined by

$L_{2 e}{ }^{+}=\left\{f:[0, \infty) \rightarrow R^{p} \mid f(t) \in L_{2}(0, T)\right.$,

for all $T \geq 0\}$

$L_{2 e}{ }^{-}=\left\{f:(-\infty, 0] \rightarrow R^{p} \mid f(t) \in L_{2}(-T, 0)\right.$,

for all $T \geq 0\}$.

Thus a function in $L_{2 e}+\left(L_{2 e}{ }^{-}\right)$is a vector-valued function defined on the positive (negative) half-line whose norm is square integrable over bounded sets.

An outline of the paper is given at the end of the next section. The main emphasis in the paper is on the presentation of results. Proofs are deleted whenever the material is easily accessible in references.

\section{Preliminaries}

We are primarily interested in optimization problems of the type

$$
\min \int_{0}^{\infty} w(x, u) d t
$$

with $w(x, \boldsymbol{u})$ given by the general quadratic form $w(x, \boldsymbol{u})=$ $\langle u, \boldsymbol{R} u\rangle+2\langle\boldsymbol{u}, \boldsymbol{C x}\rangle+\langle\boldsymbol{x}, \boldsymbol{Q} \boldsymbol{x}\rangle$ and $x$ and $u$ subject to the dynamical constraint $\dot{x}=A x+B u$ and the initial condition $x(0)=x_{0}$. We will assume without loss of generality that $R=R^{\prime}$ and $Q=Q^{\prime}$. No a priori definiteness conditions are made on the quadratic form $w(x, u)$. The results obtained thus become applicable to much more general situations than those considered in the regulator problem. As particular examples we have in mind applications to control problems with conflicting objectives $\left(\boldsymbol{C}=\mathbf{0}\right.$ and $\left.Q=\boldsymbol{C}_{1}{ }^{\prime} \boldsymbol{C}_{1}-\boldsymbol{C}_{2}{ }^{\prime} \boldsymbol{C}_{2}\right)$ to problems in which the dissipated energy $\left(\boldsymbol{\int}\langle\boldsymbol{u}, \boldsymbol{y}\rangle d t\right.$, with $y=\boldsymbol{C x}+$ $D \boldsymbol{u})$ is to be minimized and to singular problems ( $R$ singular).
As far as the terminal value of $x(t), \lim _{t \rightarrow \infty} x(t)=x_{\infty}$ is concerned, there are two cases which are of particular importance, namely, when $x_{\infty}$ is free and when $x_{\infty}=0$. Most of the results extend to the more general case in which the terminal state is required to belong to some linear manifold $H x_{\infty}=0$, but some of the details remain to be worked out. The case $x_{\infty}=0$ and $x_{\infty}$ free are fundamentally different-particularly where it concerns the boundedness of the infimum of the performance criterion.

The analytical treatment of the class of optimization problems introduced in the preceding leads to a series of matrix relations and frequency domain inequalities. Those that are important for our purposes are listed as follows. We will be interested in the case $K=K^{\prime}$.

1) The Linear Matrix Inequality (LMI):

$$
F(K)=\left[\begin{array}{cc}
A^{\prime} K+K A+Q & K B+C^{\prime} \\
B^{\prime} K+C & R
\end{array}\right] \geq 0 .
$$

2) The Quadratic Matrix Inequality (QMI):

$$
A^{\prime} K+K A-\left(K B+C^{\prime}\right) R^{-1}\left(B^{\prime} K+C\right)+Q \geq 0 .
$$

3) The Algebraic Riccati Equation (ARE):

$$
A^{\prime} K+K A-\left(K B+C^{\prime}\right) R^{-1}\left(B^{\prime} K+C\right)+Q=0 .
$$

4) The Frequency-Domain Inequality (FDI):

$$
\begin{array}{r}
H(\bar{s}, s)=R+C(I s-A)^{-1} B+B^{\prime}\left(I \bar{s}-A^{\prime}\right)^{-1} C^{\prime} \\
+B^{\prime}\left(I \bar{s}-A^{\prime}\right)^{-1} Q(I s-A)^{-1} B \geq 0 .
\end{array}
$$

It is very well known that the ARE plays a crucial role in the solution of the optimal control problem under consideration. (One often gets the impression that this equation in fact constitutes the bottleneck of all of linear system theory.) However, it is much less appreciated how the other relations enter into the theory. We hope that their role will be clarified in this paper.

The paper is organized as follows. In Section III we give a precise definition of the optimization problems considered here and of some related optimization problems. This is followed by two theorems which provide equivalent conditions for the boundedness of the infima to these problems. None of these conditions is explicit, however. This situation is remedied in Section IV where time-domain conditions are given in terms of the LMI and QMII, and frequency-domain conditions in terms of the FDI. We then turn our attention to the actual evaluation of the minima. This leads us to study the existence of solutions to the ARE. This is the subject of Section V. In Section VI we obtain a complete classification of all the solutions to the ARE. In Section VII we return to the optimization problems introduced in Section III and use the results on the ARE to derive solutions to these problems. The paper concludes with some remarks that contrast the results obtained here with the methods based on spectrum factorization ideas and with the structure of the Hamiltonian matrix. 


\section{Some Related Minimization Problems AND INEQUALITIES}

In this section we obtain some preliminary conditions for the boundedness of the infimum of the functional $\eta=\boldsymbol{S}_{0}^{\infty} w(\boldsymbol{x}, \boldsymbol{u}) d t$. This depends in a crucial way on whether $x_{\infty}$ is free or $x_{\infty}=0$. The problem is most easily approached by considering a number of related minimization problems. These are defined as follows:

$$
\begin{aligned}
& V_{f}^{+}\left(x_{0}\right)=\inf _{u \in L_{2 e^{+}}} \int_{0}^{\infty} w(x, u) d t \\
& V^{+}\left(x_{0}\right)=\inf _{u \in L_{2 e^{+}}} \int_{0}^{\infty} w(x, u) d t, \quad \text { subject to } \lim _{t \rightarrow \infty} x(t)=0 \\
& V^{-}\left(x_{0}\right)=-\inf _{u \in L_{s} e^{-}} \int_{0}^{\infty} w(x, u) d t, \\
& \text { subject to } \lim _{t \rightarrow-\infty} x(t)=0
\end{aligned}
$$$$
V_{n}+\left(x_{0}\right)=\inf _{\substack{u \in L_{2} e^{+} \\ T \geq 0}} \int_{0}^{T} w(x, \boldsymbol{u}) d t .
$$

In all of the preceding minimization problems $x$ and $u$ are constrained by the dynamical equations $\dot{x}=A x+$ $B u$ and the initial condition $x(0)=x_{0}$. It is clear that $V_{n}{ }^{+} \leq 0$ and $V_{n}{ }^{+} \leq V_{f^{+}} \leq V^{+}$. Moreover, by controllability $V_{n}{ }^{+}, V_{f}+, V^{+}<+\infty$ and $V^{-}>-\infty$.

Consider also the class of functions $V: R^{n} \rightarrow R$ which satisfies the dissipation inequality (DIE)

$$
\int_{t_{0}}^{t_{1}} w(x, u) d t+V\left(x_{1}\right) \geq V\left(x_{0}\right)
$$

for all $t_{1} \geq t_{0}$ and $x$ and $u$ satisfying $\dot{x}=A x+B u, x\left(t_{0}\right)=$ $x_{0}$, and $x\left(t_{1}\right)=x_{1}$. Assume moreover that $V$ is normalized to $V(\mathbf{0})=\mathbf{0}$. In differential form this inequality becomes

$$
\nabla_{\boldsymbol{x}} V(x) \cdot \dot{x} \geq-w(\boldsymbol{x}, \boldsymbol{u})
$$

with $\dot{x}=A x+B u$. It is a simple matter to verify, directly from the definitions, that if any of the functions $V_{f}{ }^{+}$, $V^{+}, V^{-}$, or $V_{n}^{+}$are bounded, then they satisfy the DIE. The following two theorems state a number of equivalent conditions for the boundedness of these functions.

\section{Theorem 1}

Assume that the system $\dot{x}=A x+B u$ is controllable. Then the following conditions are equivalent:

1) $\boldsymbol{S}_{0}^{T} w(x, u) d t \geq 0$, for every $T \geq 0$ and every pair $x, u$ constrained by the dynamical equations and $x(0)=0$

2) $V^{-} \leq 0$

3) $V_{f}^{+}>-\infty$;

4) $V_{n}+>-\infty$

5) There exists a function $V \leq 0$ which satisfies the DIE. Moreover, whenever any of these conditions is satisfied, then

$$
-\infty<V^{-} \leq V_{n}^{+} \leq V_{f^{+}} \leq V^{+}<+\infty .
$$

Finally, any function $V$ satisfying the DIE satisfies $V^{-} \leq V \leq V^{+}$and $V^{-} \leq V \leq V_{n}+$ if $V \leq 0$.

Proof: The proof follows the pattern:

$1) \rightarrow 2) \rightarrow 5) \rightarrow 1) \backslash \begin{aligned} & 4) \\ & 1 \\ & 3)\end{aligned}$ 1). The subcycle, 1) $\rightarrow$ 2) $\rightarrow 5) \rightarrow 1$ ) is obvious. Moreover since 1) implies $-\boldsymbol{\int}_{-\infty}^{0} w(x, u) d t \leq \boldsymbol{\int}_{0}^{T} w(x, u) d t$ whenever $\lim _{t \rightarrow-\infty} x(t)=$ 0, we obtain 1) $\rightarrow V^{-} \leq V_{n}{ }^{+} \leq V_{f}{ }^{+}$which yields 3) and 4). The implication 4 ) $\rightarrow$ 3) follows trivially and 3 ) $\rightarrow$ 1) is most easily established by contradiction.

Assume therefore that 1) is not satisfied for some $u_{0}$ and $T_{0}$. It then follows by choosing $\boldsymbol{u}(t)=\mathbf{0}$ initially that for any $T \geq T_{0}$ there exists a $u$ such that 1 ) is not satisfied. By linearity of the map $u \rightarrow x$ when $x(0)=0$; this implies that $\int_{0}^{\infty} w(x, u) d t$ can be made arbitrarily large and negative, for $x(0)=0$, and by controllability, for any $x(0)$. This establishes the contradiction. The inequalities at the end of the theorem statement are immediate.

Theorem 1 treats the case when $x_{\infty}$ is free. The case $x_{\infty}=0$ is treated in the following theorem. It may be proven in an entirely analogous manner.

\section{Theorem 2}

Assume that the system $\dot{x}=A x+B u$ is controllable. Then the following conditions are equivalent:

1) $\boldsymbol{f}_{0}^{T} w(x, u) d t$, for every $T \geq 0$ and every pair $x, u$ constrained by the dynamical equations and $x(0)=$ $x(T)=0$

2) $V^{+}>-\infty$;

3) $V-<+\infty$;

4) there exists a function $V$ which satisfies the DIE.

Moreover, whenever any of these conditions are satisfied, then

$$
-\infty<V^{-} \leq V \leq V^{+}<+\infty .
$$

Remark 1: The first condition of Theorems 1 and 2 is trivially satisfied when $w(\boldsymbol{x}, \boldsymbol{u}) \geq 0$. Consequently, no questions regarding boundedness of the infima occur in the standard regulator problem provided, as assumed here, we have controllability.

Remark 2: If, for all $x$, there exists a $u$ such that $w(\boldsymbol{x}, \boldsymbol{u}) \leq 0$, then $V_{n}{ }^{+}=V_{f}{ }^{+}$. Moreover, if there exists a feedback control law $\boldsymbol{u}(\boldsymbol{x})$ such that $w(\boldsymbol{x}, \boldsymbol{u}(\boldsymbol{x})) \leq \mathbf{0}$ and such that $\dot{x}=A x+B u(x)$ is asymptotically stable, then $V_{n}{ }^{+}=V_{f^{+}}{ }^{+}=V^{+}$and every $V$ which satisfies the DIE yields $V^{-} \leq V \leq V^{+}=V_{f}^{+}=V_{n}{ }^{+} \leq 0$.

Remark 8: Theorem 1 may be interpreted in the context of dissipative systems with $-w$ playing the role of the delivered supply rate. The functions $-V \geq 0$ which satisfy the DIE may then be interpreted as the possible storage functions with $-V_{n}{ }^{+}$and $-V$ - playing the role of the available storage and the required supply, respectively. For a formal study of these concepts, see [9]. In most of these problems the conditions of Remark 2 
hold, i.e., we can make the supply flow out of the system by suitable termination, and there is some storage left to be extracted unless $x=\mathbf{0}$.

Remark 4: Theorem 2 may be interpreted in terms of systems which appear to be dissipative when they are taken in a cyclic motion. Examples of such systems are electrical networks with $+R, \pm L, \pm C$.

Remark 5: The ideas exposed in Remarks 3 and 4 are basic tools in stability theory. If $w(x, 0) \geq 0$, then $-V$ is an obvious candidate for a Lyapunov function since the DIE states that it is nonincreasing along undriven motions. Thus dissipative systems (Theorem 1 ) suggest stability $(-V-\geq 0$ and usually by Remark 2 all the $-V \geq 0)$, and systems which appear to be dissipative when they are taken in a cycle (Theorem 2) but which are not truly dissipative $\left(-V^{-} \geq 0\right)$ suggest instability. Essentially all of the recent frequency domain stability and instability criteria [5], [6], [10] may be interpreted in the vein.

Remark 6: There may be some confusion about how to interpret $V_{f}^{+}$(and $V^{+}, V^{-}$). For purposes of infimization one may interpret the functionals as they stand, i.e., as $\inf _{u} \lim _{T \rightarrow \infty} \boldsymbol{J}_{0}^{T}$ or as $\lim _{T \rightarrow \infty} \inf _{u} \boldsymbol{S}_{0}^{T}$. It is only when considering minimizations that some care has to be exercised.

\section{Time-Domain and Frequency-Domain Conditions FOR BOUNDEDNESS OF THE INFIMA}

The results obtained in the previous section lead readily to explicit conditions for boundedness of the infima. These conditions are entirely in terms of the parameters of the system and the criterion function.

For quadratic functions $V(x)=\langle x, K x\rangle$ the DIE is equivalent to the LMI. This is easily seen by writing the DIE in its differential form: $2\langle\boldsymbol{K} x, A x+B u\rangle \geq$ $-\langle u, R u\rangle-2\langle u, C x\rangle-\langle x, Q x\rangle$.

\section{Theorem 8}

Assume that the system $\dot{x}=A x+B u$ is controllable. Then the condition $V_{f}{ }^{+}>-\infty$ is satisfied if and only if there exists a real symmetric solution $K=K^{\prime} \leq 0$ to the LMI. The condition $V^{+}>-\infty$ is satisfied if and only if there exists any real symmetric solution $K=K^{\prime}$ to the LiMI.

Proof: It is well known that the minimum of $\boldsymbol{f}_{0}^{T} w(x, \boldsymbol{u})$ $d t$ subject to $\dot{x}=A x+B u, x(0)=x_{0}$, and $x(T)$ free or $x(T)=0$ is a quadratic function of $x_{0}$. Thus the functions $V_{f}^{+}, V^{+}, V-$, and $V_{n}{ }^{+}$are, whenever they are bounded, also quadratic functions of $x_{0}$. This fact, together with the results of Theorems 1 and 2 , immediately establishes the theorem.

If we use the notation $V_{f}+(x)=\left\langle x, K_{f} x\right\rangle, V^{+}(x)=$ $\left\langle x, K^{+} x\right\rangle, V^{-}(x)=\left\langle x, K^{-} x\right\rangle$, and $V_{n}{ }^{+}=\left\langle x, K_{n}+x\right\rangle$, then we may conclude that any solution $K=K^{\prime}$ of the LMI satisfies $K^{-} \leq K \leq K^{+}$and any solution $K=K^{\prime} \leq 0$ satisfies $\boldsymbol{K}^{-} \leq \boldsymbol{K} \leq \boldsymbol{K}_{n}+\leq \mathbf{0}$.

Note that $R \geq 0$ is an obvious necessary condition for boundedness of the infima. In the case $R>0$ the LMI is equivalent to the QMI. This links the conditions of
Theorem 1 with the quadratic matrix equations of optimal control theory. The basic importance of the LMI seems to be largely unappreciated. It would be interesting to see whether or not it can be exploited in computational algorithms, for example.

Remark 7: It may be concluded from Theorem 3 that the solutions of the LMI and thus of the QMII always attain their upper and lower bounds even if we only consider nonpositive definite (or, for that matter, nonnegative definite) solutions. Note finally that the set of symmetric solutions of the LMII is closed and convex. It is also clear that if any solution $K=K^{\prime} \leq 0$ exists, then $K^{-} \leq$ 0. Interestingly, the boundedness of $V_{f}{ }^{+}$may thus be verified by evaluating $V^{-}$and hence by solving an optimization problem which has seemingly very little relation to the original problem.

Remark 8: When $R=0$, then the LMI reduces to $A^{\prime} K+$ $K A+Q \geq 0$ and $K B=-C^{\prime}$. Hence $B^{\prime} C^{\prime}=C B \geq 0$ is a necessary condition for the boundedness of inf $\eta$ when $x_{\infty}$ is free, and $B^{\prime} C^{\prime}=C B$ is a necessary condition when $x_{\infty}=\mathbf{0}$. These conditions play an important role in singular optimal control problems [26]. They are necessary conditions but far from sufficient.

We will now establish the analogue to Theorem 3 in the frequency domain.

\section{Theorem \&}

Assume that the system $\dot{x}=A x+B u$ is controllable. Then the condition $V_{f}^{+}>-\infty$ is satisfied if and only if the FDI is satisfied throughout $\operatorname{Re} s \geq 0$. The condition $\mathrm{V}^{+}>-\infty$ is satisfied if and only if the FDI is satisfied along $\operatorname{Re} s=0$.

Proof: If there exists a solution $K=K^{\prime}$ to the LMI, then

$$
\begin{aligned}
& {\left[\begin{array}{cc}
-\left(I \hat{s}-A^{\prime}\right) K-K(I s-A) & K B+C^{\prime} \\
B^{\prime} K+C & R
\end{array}\right] } \\
& \geq\left[\begin{array}{cc}
-2 \sigma K & 0 \\
0 & 0
\end{array}\right]
\end{aligned}
$$

with $s=\sigma+j \omega(\sigma, \omega \mathrm{Re})$ and $\bar{s}=\sigma-j \omega$. Postmultiplication by

$$
\left[\begin{array}{c}
\left(I_{s}-A\right)^{-1} B \\
I
\end{array}\right]
$$

and premultiplication by $\left[B^{\prime}\left(I \bar{s}-A^{\prime}\right)^{-1} \quad I\right]$ yields

$$
H(\bar{s}, s) \geq-2 \sigma B^{\prime}\left(I \bar{s}-A^{\prime}\right)^{-1} K(I s-A)^{-1} B .
$$

This shows that the frequency-domain conditions are indeed necessary conditions.

To show sufficiency, consider first the case $H(-j \omega$, $j \omega) \geq 0$. Let $u$ be a control which transfers $x(0)=0$ to $x(T)=0$, and let us assume that $u(t)=0$ and $x(t)=0$, for $t<0$ and $t>T$. Taking Fourier transforms, it follows that (whether or not $\operatorname{Re} \lambda(A)<0$ !) $X(j \omega)=(I j \omega-A)^{-1}$ $U(j \omega)$. Using Parseval's equality this implies

$$
\int_{0}^{T} w(x, u) d t \geq 0
$$


which shows that $V^{+}>-\infty$ by Theorem 2 . The sufficiency proof for the case $H(\bar{s}, s) \geq 0$ in Re $s \geq 0$ is deleted since it is rather involved.

Remark 9: Theorem 4 brings out the symmetric nature of the $x_{\infty}=0$ problem. The problem statement then does not involve any asymmetry in the time direction and hence we obtain, as expected, a frequency-domain condition along the $j \omega$ axis only. When $x_{\infty}$ is free on the other hand, the problem favors one particular time direction and we obtain a frequency-domain condition in a half-plane. This situation is not unlike that encountered when inverting a convolution operator whose kernel has the one-sided Laplace transform $g(s)$. Invertibility on $(-\infty,+\infty)$ simply requires the frequencydomain condition $|g(s)| \geq \epsilon>0$ along the $j \omega$ axis, whereas invertibility on $[0, \infty)$ requires $|g(s)| \geq \epsilon>0$ in all of $\operatorname{Re} s \geq 0$. Nyquist's criterion exploits the fact that the right half-plane inequality reduces to the $j \omega$-axis inequality coupled with an encirclement condition.

\section{The Algebraic Riccati Equation}

The results obtained in the previous section settled the question of the boundedness of the infima of the functionals which we are attempting to minimize. We now proceed toward the actual minimization. It is hardly surprising that this road leads by way of the ARE. Thus the existence of an optimum is most easily established via the QMI, whereas the actual optimum is related to those matrices which satisfy this inequality with equality.

Remark 10: One may be lead to wonder in what sense the solutions to the ARE are "special" solutions of the LMI. The solutions of the ARE are boundary solutions of the LMI in the sense that they make the matrix $F(K)$ of minimal rank. It would be of interest to investigate if and how the solutions of the LMI which make $F(K)$ of minimal rank are related to the solution of the singular optimal control problems which arise when $R$ is singular.

We first establish a fundamental lemma.

\section{Lemma 1}

Let $\boldsymbol{K}$ be a real symmetric solution to the ARE. Then $K$ satisfies the identity

$$
\begin{aligned}
R+C & (I s-A)^{-1} B+B^{\prime}\left(I z-A^{\prime}\right)^{-1} C^{\prime}+B^{\prime} \\
& \left(I z-A^{\prime}\right)^{-1} Q(I s-A)^{-1} B=(I+T(z))^{\prime} R \\
& (I+T(s))-(z+s) B^{\prime}\left(I z-A^{\prime}\right)^{-1} K(I z-A)^{-1} B
\end{aligned}
$$

where

$$
T(s)=R^{-1}\left(B^{\prime} K+C\right)(I s-A)^{-1} B .
$$

Thus in particular $H(-j \omega, j \omega) \geq 0$, for all real $\omega$.

Proof: The proof involves standard algebraic manipulations and is deleted.

The inequality stated in Lemma 1 and Theorem 4 show that the condition $H(-j \omega, j \omega) \geq 0$ is a necessary condition for the existence of a real symmetric solution to the ARE. This condition turns out to be sufficient as well. The proof of this fact is rather involved and requires a number of intermediate results which are of intrinsic importance. It is thus most convenient to establish this result with the aid of a series of auxiliary lemmas.

We will assume throughout Sections V and VI that $R$ $\geq 0$. As is well known, one may then reduce the minimization problem with the criterion function $\int w(x, u) d t$ to a similar one with $R=I$ and $C=0$. We will therefore assume this to be the case throughout the preliminary analysis and show in the proof of the general theorem how to extend the results to the case $R \neq I$ and /or $\boldsymbol{C} \neq 0$.

The algebraic Riccati equation considered in the following lemmas thus reads

$$
A^{\prime} K+K A-K B B^{\prime} K+Q=0, \quad\left(\mathrm{ARE}^{\prime}\right) .
$$

We begin with a result on uniqueness and a result which orders the solutions to the ARE.

\section{Lemma \&}

There exists at most one real symmetric solution to ARE' having the property that $\operatorname{Re} \lambda\left(A-B B^{\prime} K\right)<0$ $(>0)$.

Proof: See [3, p. 150].

\section{Lemma 3}

Let $K_{1}$ be a real symmetric solution of the ARE

$$
A^{\prime} K_{1}+K_{1} A-K_{1} B B^{\prime} K_{1}+Q_{1}=0
$$

with $Q_{1}=Q_{1}{ }^{\prime}$, and assume that $\operatorname{Re} \lambda\left(A-B B^{\prime} K_{1}\right)<0$ $(>0)$. Then any real symmetric solution $K_{2}$ of the ARE

$$
A^{\prime} K_{2}+K_{2} A-K_{2} B B^{\prime} K_{2}+Q_{2}=0
$$

with $Q_{2}=Q_{2}{ }^{\prime}$ and $Q_{1} \geq Q_{2}$ satisfies $K_{1} \geq K_{2}\left(K_{1} \leq K_{2}\right)$.

Proof: Let $\Delta Q=Q_{1}-Q_{2}$ and $\Delta K=K_{1}-K_{2}$. Subtracting the two preceding $\mathrm{ARE}$ and reordering terms yields

$$
\begin{aligned}
\left(A-B B^{\prime} K_{1}\right)^{\prime} \Delta K+\Delta K\left(A-B B^{\prime} K_{1}\right) & \\
& =-\Delta K B B^{\prime} \Delta K-\Delta Q .
\end{aligned}
$$

Consequently, since $\operatorname{Re} \lambda\left(A-B B^{\prime} K_{1}\right)<0$,

$$
\begin{array}{r}
\Delta K=\int_{0}^{\infty} \exp \left[\left(A-B B^{\prime} K_{1}\right)^{\prime} t\right]\left(\Delta K B B^{\prime} \Delta K+\Delta Q\right) \\
\cdot \exp \left[\left(A-B B^{\prime} K_{1}\right) t\right] d t .
\end{array}
$$

Thus $\Delta K \geq 0$ as claimed. The case $\operatorname{Re} \lambda\left(A-B B^{\prime} K_{1}\right)>0$ is proved in a similar way.

This lemma yields as an immediate consequence the following ordering on the solutions of a particular ARE. Assume that ${ }^{1} K^{+}$and $K^{-}$are real symmetric solutions of the ARE' having the property that $\operatorname{Re} \lambda\left(A-B B^{\prime} K^{+}\right)<0$ and $\operatorname{Re} \lambda\left(A-B B^{\prime} K^{-}\right)>0$, respectively, and let $K$ be any other symmetric solution. Then $K^{-} \leq K \leq K^{+}$.

${ }^{1}$ The notation $K^{+}$and $K^{-}$is at this point somewhat ambiguous since we already introduced a similar notation in Section IV. This ambiguity is actually deliberate since it will turn out that they are the same matrices as those introduced in Section IV. 
Note that Lemma 3 also establishes that (assuming they exist) $K^{+}$is monotone nondecreasing with increasing $Q$, whereas $K^{-}$is monotone nonincreasing with increasing $Q$. Consequently, $\boldsymbol{K}^{+}-\boldsymbol{K}^{-}$is monotone nondecreasing with increasing $Q$.

We now treat the case in which $Q$ is nonnegative definite. This is the classical case studied in the standard regulator problem.

\section{Lemma 4}

Assume that the system $\dot{x}=A x+B u ; y=C_{1} x$ is controllable and observable. Then there exists a real symmetric solution to the $\mathrm{ARE}^{\prime}$ with $Q=C_{1}{ }^{\prime} C_{1}$, which has the property that $\operatorname{Re} \lambda\left(A-B B^{\prime} K\right)<0(>0)$. Moreover, such a solution is unique and has the additional property that $K=K^{\prime}>0(<0)$. In fact, it is also the only solution in the cone ${ }^{2}$ of symmetric positive (negative) semidefinite matrices.

Proof: This lemma constitutes a fundamental result in modern control and filtering theory. For complete expositions, we refer the reader to Kalman [1], Kleinman [11], or Brockett [3, p. 151].

Remark 11: There have been a number of recent papers [12]-[14] treating extensions of Lemma 4 to noncontrollable and/or nonobservable systems. Particularly the results in [14] are of interest as a comparison with the results of Sections VI and VII.

The next lemma treats in a sense the other extreme, namely when $Q$ is negative semidefinite. Although the treatment of the resulting optimal control problem is not so well known in optimal control circles, it is, however, a basic tool in stability theory. As is well known, a solution will not always exist in this case. The relevant condition is given in Lemma 5.

\section{Lemma 5}

Assume that the system $\dot{x}=A x+B u ; y=C_{2} x$ is controllable and observable and that $\operatorname{Re} \lambda(A)<0(>0)$. Then there exists a real symmetric solution to the ARE' with $Q=-C_{2}{ }^{\prime} C_{2}$ having the property $\operatorname{Re} \lambda\left(A-B B^{\prime} K\right)$ $\leq 0(\geq 0)$ if and only if

$$
\begin{aligned}
I-B^{\prime}\left(-I j \omega-A^{\prime}\right)^{-1} C_{2}{ }^{\prime} C_{2}(I j \omega-A)^{-1} B & \geq 0, \\
& \text { for all real } \omega .
\end{aligned}
$$

Moreover such a solution is unique and has the additional property that $K=K^{\prime}<\mathbf{0}$.

Proof: See [3, p. 167) and [6].

Remark 12: The frequency-domain condition in Lemma 5 is actually equivalent to $H(\bar{s}, s) \geq 0$ in $\operatorname{Re} s \geq 0$ since $\operatorname{Re} \lambda(A)<0$.

Remark 13: It is worthwhile to contrast the standard result of Lemma 4 with that of Lemma 5. Both in Lemmas

2 The cone of symmetric positive (negative) semidefinite matrices simply refers to the set of all such matrices.
4 and 5 we obtain many solutions to the ARE, but only one of them has the additional property $\operatorname{Re} \lambda\left(A-B B^{\prime} K\right)$ $\leq 0(\geq 0)$. This solution turns out to be the maximum (minimum) solution. However, in Lemma 4 the maximum solution is positive definite, the minimum solution is negative definite, and all remaining solutions are indefinite, whereas in Lemma 5 the maximum solution is negative definite, and consequently all solutions are negative definite.

Remark 14: The importance of Lemma 5 in stability theory may be explained in a few words as follows. Consider the nonlinear time-varying differential equation $\dot{x}=A x-B f(C x, t)$ and assume that $A, B, C$ satisfy the conditions of Lemma 5 . Let $K=K^{\prime}<0$ be a solution of the $\mathrm{ARE}^{\prime}$, with $Q=-\boldsymbol{C}^{\prime} \boldsymbol{C}$, and consider the derivative of $V(x)=-\langle x, K x\rangle$ along solutions to the preceding differential equation. Then

$$
\dot{V}(x)=-\left\|B^{\prime} K x-f(C x, t)\right\|^{2}-\left(\|C x\|^{2}-\|f(C x, t)\|^{2}\right) .
$$

This then establishes the stability of the differential equation if $\|f(y, t)\| \leq\|y\|$, for all $y$ and $t$. For a conceptualization of this procedure see [15].

We are now ready to state the main result of this section. It consists of a necessary and sufficient condition for existence of solutions to the ARE.

\section{Theorem 5}

Assume that the system $\dot{x}=A x+B u$ is completely controllable. Then the following are true.

1) Existence: The ARE has a real symmetric solution if and only if $H(-j \omega, j \omega) \geq 0$, for all real $\omega$. There is then always exactly one such solution, denoted by $K^{+}$, which has the additional property that $\operatorname{Re} \lambda\left(A^{+}\right) \leq 0$, with $A^{+}=A-B R^{-1}\left(B^{\prime} K^{+}+C\right)$ and exactly one such solution, denoted by $\boldsymbol{K}^{-}$, which has the additional property that $\operatorname{Re} \lambda\left(A^{-}\right) \geq 0$, with $A^{-}=A-B R^{-1}\left(B^{\prime} K^{-}+C\right)$.

2) Ordering: Any other real symmetric solution $K$ is ordered with respect to these special solutions by the inequality $K^{-} \leq K \leq K^{+}$.

3) Strict Inequality: One of the strict inequalities

$$
H(-j \omega, j \omega) \geq \epsilon B^{\prime}\left(-I j \omega-A^{\prime}\right)^{-1}(I j \omega-A)^{-1} B,
$$

for some $\epsilon>0$

$$
\begin{aligned}
& \operatorname{Re} \lambda\left(A^{+}\right)<0 \\
& \operatorname{Re} \lambda\left(A^{-}\right)>0 \\
& \boldsymbol{K}^{+}-\boldsymbol{K}^{-}>\mathbf{0}
\end{aligned}
$$

implies the others, in which case

$$
\begin{aligned}
K^{+}-K^{-} & =\left(\int_{0}^{\infty} \exp \left(A^{+} t\right) B B^{\prime} \exp \left(A^{+\prime} t\right) d t\right)^{-1} \\
& =\left(\int_{-\infty}^{0} \exp \left(A^{-} t\right) B B^{\prime} \exp \left(A^{-\prime} t\right) d t\right)^{-1} .
\end{aligned}
$$

Proof: Assume first that $C=0$ and $R=I$.

1) Existence: The frequency-domain condition is necessary by Lemma 1 . To show that it is also sufficient 
let $Q=C_{1}{ }^{\prime} C_{1}-C_{2}{ }^{\prime} C_{2}$, with $C_{1}$ and $C_{2}$ invertible. Such a decomposition clearly exists since $Q=r I-(r I-Q)$, with $r$ sufficiently large, defines one such possibility. Let $K_{1}$ now be the positive definite solution to the ARE

$$
A^{\prime} K_{1}+K_{1} A-K_{1} B B^{\prime} K_{1}+C_{1}^{\prime} C_{1}=0 .
$$

By Lemma $4, K_{1}$ indeed exists and is such that $A_{1}=A-$ $B B^{\prime} K_{1}$ satisfies $\operatorname{Re} \lambda\left(A_{1}\right)<0$. Let $\Delta K=K-K_{1}$. The equation in $\Delta K$ then becomes

$$
A_{1}{ }^{\prime} \Delta K+\Delta K A_{1}-\Delta K B B^{\prime} \Delta K-C_{2}{ }^{\prime} C_{2}=0 .
$$

To show that this has a solution, let us compute

$$
I-B^{\prime}\left(-I j \omega-A_{1}{ }^{\prime}\right)^{-1} C_{2}{ }^{\prime} C_{2}\left(I j \omega-A_{1}\right)^{-1} B .
$$

Notice first that

$$
\begin{aligned}
C_{2}\left(I s-A+B B^{\prime} K_{1}\right)^{-1} B= & C_{2}(I s-A)^{-1}\left[I+B B^{\prime} K_{1}\right. \\
& \left.\cdot(I s-A)^{-1}\right]^{-1} B \\
= & C_{2}(I s-A)^{-1} B\left[I+B^{\prime} K_{1}\right. \\
& \left.\cdot(I s-A)^{-1} B\right]^{-1} .
\end{aligned}
$$

From the definition of $\boldsymbol{K}_{1}$ and Lemma 1 we also have that

$I+B^{\prime}\left(-I s-A^{\prime}\right)^{-1} C_{1}{ }^{\prime} C_{1}(I s-A)^{-1} B$

$=\left[I+B^{\prime} K_{1}(-I s-A)^{-1} B\right]^{\prime}\left[I+B^{\prime} K_{1}(I s-A)^{-1} B\right]$.

Now, since by assumption

$$
\begin{aligned}
I+B^{\prime}(-I j \omega- & \left.A^{\prime}\right)^{-1} C_{1}{ }^{\prime} C_{1}(I j \omega-A)^{-1} B- \\
& B^{\prime}(-I j \omega-A)^{-1} C_{2}{ }^{\prime} C_{2}(I j \omega-A)^{-1} B \geq 0
\end{aligned}
$$

it follows that

$$
\begin{aligned}
I- & \left\{C_{2}(-I j \omega-A)^{-1} B\left[I+B^{\prime} K_{1}(-I j \omega-A)^{-1} B\right]^{-1}\right\}^{\prime} \\
& \cdot\left\{C_{2}(I j \omega-A)^{-1} B\left[I+B^{\prime} K_{1}(I j \omega-A)^{-1} B\right]^{-1}\right\} \geq 0 .
\end{aligned}
$$

Consequently,

$$
I-B^{\prime}\left(-I j \omega-A_{1}{ }^{\prime}\right)^{-1} C_{2}{ }^{\prime} C_{2}\left(I j \omega-A_{1}\right)^{-1} B \geq 0 .
$$

We may now apply Lemma 6 to the ARE in $\Delta K$. Consequently, there exists a unique solution $\Delta K$ such that $\operatorname{Re} \lambda\left(A_{1}-B B^{\prime} \Delta K\right)=\operatorname{Re} \lambda\left(A-B B^{\prime} K\right) \leq 0$. This shows the existence and uniqueness of $K^{+}$. Existence and uniqueness of $K^{-}$follows by analogy.

2) Ordering: The ordering relationships follow from Lemma 3 provided $\operatorname{Re} \lambda\left(A^{+}\right)<0$ and $\operatorname{Re} \lambda\left(A^{-}\right)>0$. They may be proven by a continuity argument whenever these inequalities are not strictly satisfied.

3) Strict Inequalities: Since they satisfy the $\mathrm{ARE}^{\prime}$, it follows that $K^{+}$and $K^{-}$satisfy

$$
\begin{aligned}
A^{+\prime}\left(K^{+}-K^{-}\right)+\left(K^{+}-K^{-}\right) A^{+}=-\left(K^{+}-K^{-}\right) \\
\\
\cdot B B^{\prime}\left(K^{+}-K^{-}\right) \\
A^{-\prime}\left(K^{+}-K^{-}\right)+\left(K^{+}-K^{-}\right) A^{-}=\left(K^{+}-K^{-}\right) \\
\cdot B B^{\prime}\left(K^{+}-K^{-}\right) .
\end{aligned}
$$

Thus from a sharpened version of Lyapunov's theorem which exploits controllability it follows that $K^{+}-K^{-}>0$ implies $\operatorname{Re} \lambda\left(\boldsymbol{A}^{+}\right)<0$ and $\operatorname{Re} \lambda\left(\boldsymbol{A}^{-}\right)>0$.
To prove the converse, let $\Delta+K=K^{+}-K$ and $\Delta-K=$ $K^{-}-K$. The equations satisfied by $\Delta^{+} K$ and $\Delta^{-} K$ are

$$
\begin{aligned}
& A^{+\prime} \Delta^{+} K+\Delta^{+} K A^{+}=-\Delta^{+} K B B^{\prime} \Delta^{+} K \\
& A^{-\prime} \Delta^{-} K+\Delta-K A^{-}=-\Delta^{-} K B B^{\prime} \Delta^{-} K \text {. }
\end{aligned}
$$

Assuming now that $\operatorname{Re} \lambda\left(A^{+}\right)<0$, we notice (since the equation in $\left(\Delta^{+} K\right)^{-1}$ is linear) that the first of these equations has exactly one invertible solution (in addition to many noninvertible solutions). In fact, that invertible solution equals

$$
\left(\int_{0}^{\infty} \exp \left(A^{+} t\right) B B^{\prime} \exp \left(A^{+\prime} t\right) d t\right)^{-1}
$$

This solution leads to a solution of the Riccati equation, say, $\tilde{K}$. A simple rearrangement of terms shows that $\left(\boldsymbol{A}-\boldsymbol{B} \boldsymbol{B}^{\prime} \tilde{K}\right)^{\prime}\left(\boldsymbol{K}^{+}-\tilde{K}\right)+\left(\boldsymbol{K}^{+}-\tilde{K}\right)\left(A-B B^{\prime} \tilde{K}\right)=$ $\left(K^{+}-\tilde{K}\right) B B^{\prime}\left(K^{+}-\tilde{K}\right)$ which, since $K^{+}-\tilde{K}>0$, shows that $\operatorname{Re} \lambda\left(A-B B^{\prime} \tilde{K}\right)>0$ and thus that $\tilde{K}=K^{-}$. Hence $\operatorname{Re} \lambda\left(A^{+}\right)<0$ implies that $K^{+}>K^{-}$as claimed. That $\operatorname{Re} \lambda\left(A^{-}\right)>0$ implies $K^{+}>K^{-}$follows again by analogy.

To prove the frequency-domain condition, consider the ARE

$$
A^{\prime} K_{\epsilon}+K_{\epsilon} A-K_{\epsilon} B B^{\prime} K_{\epsilon}+Q+\epsilon I=0 .
$$

It follows from Lemma 3 that whenever they exist, $K_{\epsilon_{1}}{ }^{+}$ $<K_{\epsilon_{2}}+$ when $\epsilon_{1}<\epsilon_{2}$. This implies by continuity that $K^{-}$ $<K^{+}$is equivalent to the frequency-domain condition in the theorem statement.

Consider now the general ARE. This equation may be reduced to one of the type ARE' if we make the substitutions $A-B R^{-1} C \rightarrow A_{1}, B R^{-1 / 2} \rightarrow B_{1}$, and $-C^{\prime} R^{-1} C+$ $Q \rightarrow Q_{1}$. A series of straightforward manipulations shows that the frequency-domain condition

$$
I+B_{1}{ }^{\prime}\left(-I j \omega-A_{1}{ }^{\prime}\right) Q_{1}\left(I j \omega-A_{1}\right)^{-1} B_{1} \geq 0
$$

is equivalent to the condition $H(-j \omega, j \omega) \geq 0$. The result then follows from the case $R=I, C=0$ treated in the preceding.

Remark 15: The solutions $\boldsymbol{K}^{+}$and $\boldsymbol{K}^{-}$, which play the special roles stated in Theorem $\tilde{5}$, have a simple behavior versus changes in $Q$ and $R$. Indeed $K^{+}$decreases as $Q$ and $R$ decrease, and $K^{-}$increases as $Q$ and $R$ decrease. This may quite easily be shown (and incidentally, follows trivially from the optimal control interpretation of Section VII). Consequently, the "gap" $K^{+}-K^{-}$decreases with decreasing $Q$ and $R$. Fig. 1 illustrates the behavior of $K^{+}$and $K^{-}$under changes in $Q$. The solution $K^{+}$decreases as $Q$ decreases, whereas $K^{-}$increases as $Q$ decreases. Consequently, at some value of $Q, K^{+}-K^{-}$ ceases to be positive definite and is merely positive semidefinite. This is also the point where solutions cease to exist and where the FDI in the statement of Theorem 5 ceases to be satisfied. Fig. 1 completes, as far as the extreme solutions to the ARE are concerned, a similar picture obtained in [16] for the case where $Q$ is definite. It is, however, of no help in giving insight or ways of 


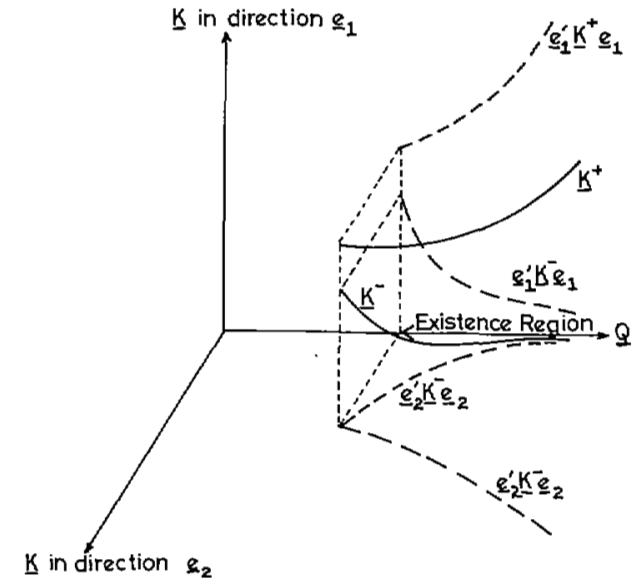

Fig. 1. Behavior of $K^{+}$and $K^{-}$as function of $Q$.

computing the intermediate solutions. The next section of this paper is concerned with such a classification.

\section{Example 1}

Consider the scalar equation $k^{2}-2 \alpha k-\lambda=0$. By Theorem 5 this equation has a real solution if and only if

$$
1+\frac{\lambda}{\alpha^{2}+\omega^{2}} \geq 0, \quad \text { for all real } \omega .
$$

This happens when $\alpha^{2}+\lambda \geq 0$, which is the familiar discriminant condition for quadratic equations.

\section{A Classification of All Solutions to the ARE}

It was shown in the previous section that (given solutions exist) the set of real symmetric solutions to the ARE always attains its maximum $K^{+}$and its minimum $K^{-}$. Theorem 5 provided a necessary and sufficient frequency-domain condition for existence and established moreover that $K^{+}$and $K^{-}$are the unique solutions having the additional property $\operatorname{Re} \lambda\left(A-B R^{-1}\left(B^{\prime} K^{+}+C\right)\right) \leq$ 0 and $\operatorname{Re} \lambda\left(A-B R^{-1}\left(B^{\prime} K^{-}+C\right)\right) \geq 0$, respectively. We now set out to study the other solutions. It will turn out that these are simply certain combinations of $K^{+}$and $K^{-}$.

Recall the following notation: let $K^{+}$and $K^{-}$be defined as shown in the preceding. Then $A^{+}$and $A^{-}$will denote $A-B R^{-1}\left(B^{\prime} K^{+}+C\right)$ and $A-B R^{-1}\left(B^{\prime} K^{-}+\right.$ $C)$, respectively. The matrix $\Delta=K^{+}-K^{-}$will be called the gap associated with the ARE. Note that Re $\lambda\left(A^{+}\right) \leq 0$ and $\operatorname{Re} \lambda\left(A^{-}\right) \geq 0$, and that $\Delta \geq 0$, with strict inequalities holding simultaneously. Let $\boldsymbol{\Delta}^{+} K=$ $K^{+}-K$ and $\boldsymbol{\Delta}^{-} \boldsymbol{K}=K^{-}-K$. Then $\boldsymbol{\Delta}^{+} \boldsymbol{K} \geq \mathbf{0}$ and $\Delta-K \leq 0$.

Let $\boldsymbol{M}$ be an $(n \times n)$ matrix, and let $S$ be a subspace of $R^{n}$. Then $S$ is said to be invariant if $M x \in S$, for all $x \in S$. In particular for any $(n \times n)$ matrix $M$ with $n^{+}$eigenvalues with negative real parts, $n^{0}$ eigenvalues with zero real parts, and $n^{-}$eigenvalues with positive real parts, one may define the invariant subspaces $\mathcal{L}^{+}(\boldsymbol{M}), \mathfrak{L}^{0}(\boldsymbol{M})$, and $\mathcal{L}^{-}(\boldsymbol{M})$, respectively, spanned by the corresponding (generalized) eigenvectors. The notation introduced in the preceding will be used throughout, and the dependence on the matrix $M$ will be deleted whenever there is no possibility of confusion.

We will now prove a lemma which is of crucial importance in least squares optimal control. It shows one how to "complete the square" with the aid of the ARE. This lemma is consistently exploited, for example, in [3]. It provides in our opinion the most direct and most elementary way of attacking least squares problems.

\section{Lemma 6}

Let $K$ be a real symmetric solution to the ARE and let $\eta=\int_{0}^{T} w(x, u) d t$, with $x$ and $u$ related through the differential equation $\dot{x}=A x+B u$ and with $x(0)=x_{0}$ and $x(T)=x_{r}$. Then $\eta=\int_{0}^{T}\left\|\boldsymbol{u}+\boldsymbol{R}^{-1}\left(B^{\prime} K+C\right) x\right\|_{R^{2}}$ $+\left\langle x_{0}, K x_{0}\right\rangle-\left\langle x_{T}, K x_{T}\right\rangle$.

Proof: Differentiation by parts yields

$$
\begin{aligned}
\boldsymbol{x}_{0}{ }^{\prime} K x_{0}-x_{T}{ }^{\prime} \boldsymbol{K} x_{T} & =-\int_{0}^{T} \frac{d}{d t}\left(x^{\prime} \boldsymbol{K} x\right) d t \\
& =-\int_{0}^{T}\left[\boldsymbol{x}^{\prime}\left(\boldsymbol{A}^{\prime} \boldsymbol{K}+\boldsymbol{K} \boldsymbol{A}\right) \boldsymbol{x}+2 \boldsymbol{u}^{\prime} \boldsymbol{B}^{\prime} \boldsymbol{K} \boldsymbol{x}\right] d t .
\end{aligned}
$$

Using the ARE, the integrand becomes

$$
\begin{aligned}
x^{\prime} & {\left[\left(K B+C^{\prime}\right) R^{-1}\left(B^{\prime} K+C\right)-Q\right] x+2 u^{\prime} B^{\prime} K x } \\
= & \left\|u+R^{-1}\left(B^{\prime} K+C\right) x\right\|_{R^{2}}-\|u\|_{R^{2}} \\
& -2 u^{\prime} C x-x^{\prime} Q x .
\end{aligned}
$$

Upon substitution in the preceding integral, this yields the desired result.

The results which follow are much easier to express when the strict inequality $\Delta>0$ is assumed to hold. We will therefore concentrate on this case since the results in the general case are pretty much the same. The modifications to treat the case in which the gap $\boldsymbol{\Delta}=\boldsymbol{K}^{+}-\boldsymbol{K}^{-}$ is merely positive semidefinite are outlined in Remark 19. The following lemma indicates in what sense an arbitrary solution of the ARE is a combination of $\mathrm{K}^{+}$ and $K^{-}$.

\section{Lemma 7}

Let $K$ be a real symmetric solution to the ARE. Then $\left(K^{+}-K\right) x=0$, for $x \in \mathscr{L}^{+}\left(A-B R^{-1}\left(B^{\prime} K+C\right)\right)$ and $\left(K-K^{-}\right) x=0$, for $x \in \mathcal{L}^{-}\left(A-B R^{-1}\left(B^{\prime} K+C\right)\right)$. Consequently, $\left(A-B R^{-1}\left(B^{\prime} K+C\right)\right) x=A^{+} x$, for $x \in \mathcal{L}^{+}\left(A-B R^{-1}\left(B^{\prime} K+C\right)\right)$ and $\left(A-B R^{-1}\left(B^{\prime} K+\right.\right.$ C)) $x=A^{-} x$, for $x \in \mathcal{L}^{-}\left(A-B R^{-1}\left(B^{\prime} K+C\right)\right)$.

Proof: Let

$$
\eta=\int_{0}^{\infty} w(x, u) d t
$$

subject to the constraints

$$
\begin{aligned}
\dot{x} & =A x+B u \\
x(0) & =x_{0} \in \mathscr{L}^{+}\left(A-B R^{-1}\left(B^{\prime} K+C\right)\right) .
\end{aligned}
$$


Using the feedback control law $u=-R^{-1}\left(B^{\prime} K+C\right) x$, we see that by Lemma 6

$$
\eta=x_{0}{ }^{\prime} K x_{0}=x_{0}{ }^{\prime} K^{+} x_{0}+\int_{0}^{\infty}\left\|R^{-1} B^{\prime}\left(K-K^{+}\right) x\right\|_{R} d t
$$

Thus $x_{0}{ }^{\prime} K x_{0} \geq x_{0}{ }^{\prime} K^{+} x_{0}$. Since by Theorem $5, x_{0}{ }^{\prime} K x_{0} \leq$ $x_{0}{ }^{\prime} K^{+} x_{0}$, it follows that $x_{0}{ }^{\prime}\left(K^{+}-K\right) x_{0}=0$, for $x_{0} \in$ $\mathcal{L}^{+}\left(A-B R^{-1}\left(B^{\prime} K+C\right)\right)$. Since $K^{+}-K \geq 0$, this indeed implies that $\left(K^{+}-K\right) x=0$, for $x \in \mathscr{L}^{+}(A-$ $\left.B R^{-1}\left(B^{\prime} K+C\right)\right)$. The equality $\left(A-B R^{-1}\left(B^{\prime} K+C\right)\right) x$ $=A^{+} x$ is an immediate consequence of this, and the analogous relationship for $x \in \mathcal{L}^{-}\left(A-B R^{-1}\left(B^{\prime} K+C\right)\right)$ may be proven in a similar fashion.

In the next lemma we assume that $\Delta>0$ and bring out some important geometrical relationships between $\mathrm{A}^{+}$and $\mathrm{A}^{-}$.

\section{Lemma 8}

Assume that the system $\dot{x}=A x+B u$ is controllable and that $\Delta=K^{+}-K^{-}>0$. Then $A^{+}$and $-A^{-^{\prime}}$ are similar matrices. In fact, $A^{+}=-\Delta^{-1} A^{-1} \Delta$. Moreover to every invariant subspace $S_{1}$ of $A^{+}$, there corresponds an invariant subspace $S_{2}=\Delta^{-1} S_{1}{ }^{\perp}$ of $A^{-}$, and $S_{1} \oplus S_{2}=$ $R^{n}$. Let $P$ be the projection operator which uniquely decomposes $x$ into $x=P x+(I-P) x$, where $P x \in S_{1}$ and $(I-P) x \in S_{2}$. Then $P$ satisfies the equations $A^{+} \boldsymbol{P}=$ $P A^{+} \boldsymbol{P}, P A^{-}=P A^{-} \boldsymbol{P}$, and $\boldsymbol{P}=\boldsymbol{\Delta}^{-1} \boldsymbol{P}^{\prime} \boldsymbol{\Delta}$. Conversely, if a projection $\boldsymbol{P}$ satisfies the equations $\boldsymbol{A}^{+} \boldsymbol{P}=\boldsymbol{P} A^{+} \boldsymbol{P}$ (or $\left.P_{A^{-}}=P A^{-} \boldsymbol{P}\right)$ and $\boldsymbol{P}=\boldsymbol{\Delta}^{-1} \boldsymbol{P}^{\prime} \boldsymbol{\Delta}$, then $S_{1}$, the range of $\boldsymbol{P}$, is invariant with respect to $\boldsymbol{A}^{+}, S_{2}$, the range of $\boldsymbol{I}-\boldsymbol{P}$, is invariant with respect to $A^{-}$, and $S_{2}=\Delta^{-1} S_{1}{ }^{\perp}$.

Proof: Since $A^{+\prime} \Delta+\Delta A^{+}=-\Delta B R^{-1} B^{\prime} \Delta$ and $A^{-}=A^{+}+B R^{-1} B^{\prime} \Delta$, it follows that $A^{+^{\prime} \Delta}+\Delta A^{-}=0$. The invariance of $\boldsymbol{\Delta}^{-1} S_{1}{ }^{\perp}$ follows immediately from this similarity relation. Consider now $S_{1} \oplus S_{2}$ and assume that $x \in S_{1} \cap S_{2}$. Then $\langle\Delta x, x\rangle=\mathbf{0}$ which implies $x=\mathbf{0}$. Thus $S_{1} \oplus S_{2}=R^{n}$. Consider now the equations in $P$. The first states that $S_{1}$ is invariant with respect to $A^{+}$; the second states that $S_{2}$ is invariant with respect to $A^{-}$; and the third one states that $S_{2}=\Delta^{-1} S_{1}{ }^{\perp}$. The second part of the lemma is thus simply a restatement of the first part.

The theorem which follows is the main result of this section and shows how to generate all the solutions to the ARE.

\section{Theorem 6}

Assume that the system $\dot{x}=A x+B u$ is completely controllable and that there exist real symmetric solutions to the ARE. Assume moreover that $\Delta=K^{+}-K^{-}>\mathbf{0}$. Then every real symmetric solution of the (ARE) may be decomposed as

$$
\begin{array}{r}
K=K^{+P}+K^{-(I-P)} \\
=K^{+}-\Delta(I-P)=K^{-}+\Delta P
\end{array}
$$

where $\boldsymbol{P}$ and $\boldsymbol{I}-\boldsymbol{P}$ are projections onto the invariant subspaces $S_{1}$ and $S_{2}=\Delta^{-1} S_{1}^{\perp}$ of $A^{+}$and $A^{-}$, respectively. Conversely to every invariant subspace $S_{1}$, of $A^{+}$there corresponds an invariant subspace $S_{2}=\Delta^{-1} S_{1}{ }^{\perp}$ of $A^{-}$ and a solution

$K=K^{+} P+K^{-}(I-P)$

$$
=K^{+}-\Delta(I-P)=K^{-}+\Delta P
$$

with $P$ and $I-P$ projections onto $S_{1}$ and $S_{2}$, respectively. Moreover,

$$
\begin{aligned}
& \mathfrak{L}^{+}\left(A-B R^{-1}\left(B^{\prime} K+C\right)\right)=S_{1} \\
& \mathcal{L}^{-}\left(A-B R^{-1}\left(B^{\prime} K+C\right)\right)=S_{2} .
\end{aligned}
$$

Proof: Let $K$ be a real symmetric solution. By Theorem 5 , $\operatorname{Re} \lambda\left(A^{+}\right) \neq 0$. Consequently, $\operatorname{Re} \lambda\left(A-B R^{-1}\left(B^{\prime} K+\right.\right.$ C)) $\neq 0$. By Lemma $7, K x=K^{+} x$, for $x \in \mathscr{L}^{+}(A-$ $\left.B R^{-1}\left(B^{\prime} K+C\right)\right)$ and $K x=K^{-} x$, for $x \in \mathcal{L}^{-}\left(A-B R^{-1}\right.$ $\left(B^{\prime} K+C\right)$ ). Since $\mathscr{L}^{+}$and $\mathscr{L}^{-}$are invariant with respect to $A-B R^{-1}\left(B^{\prime} K+C\right)$ ), they are also invariant with respect to $A^{+}$and $A^{-}$, respectively. Thus if $P$ is the projection which decomposes $x$ as $x=P x+(I-P) x$, with $P x \in \mathscr{L}^{+}$and $(I-P) x \in \mathscr{L}^{-}$, it follows that $\boldsymbol{\Delta}^{+} \boldsymbol{K}=$ $K^{+}-K=\Delta(I-P)$. The projection $P$ satisfies $A^{+} P=$ $\boldsymbol{P A}+\boldsymbol{P}$ and, since $K$ is symmetric, $\Delta \boldsymbol{P}=\boldsymbol{P}^{\prime} \boldsymbol{\Delta}$. Thus by Lemma 8 every solution may indeed be decomposed as claimed in the theorem.

Conversely, assume that $P$ is a projection which satisfies $\boldsymbol{A}^{+} \boldsymbol{P}=\boldsymbol{P} \boldsymbol{A}^{+} \boldsymbol{P}$ and $\boldsymbol{P} \boldsymbol{A}^{-}=\boldsymbol{P A}-\boldsymbol{P}$. The difference $\boldsymbol{\Delta}^{+} \boldsymbol{K}=$ $K^{+}-K$ is governed by the equation $A^{{ }^{+} \Delta^{+}} \boldsymbol{K}+\Delta^{+} K$ $A^{+}=-\Delta^{+} \boldsymbol{K} B R^{-1} B^{\prime} \Delta^{+} \boldsymbol{K}$. Thus in order for $\boldsymbol{\Delta}^{+} \boldsymbol{K}=$ $\Delta(I-P)$ to be a solution we need that

$A^{+\prime} \Delta(I-P)+\Delta(I-P) A^{+}=$

$$
-(I-P) B R^{-1} B^{\prime} \Delta(I-P) .
$$

Since $\Delta^{-1} A^{+\prime} \Delta=-A^{-}$, this requires $-A^{-}(I-P)+$ $(I-P) A^{+}=(I-P) B R^{-1} B^{\prime} \Delta(I-P)$, or, since $A^{-}$ $(I-P)=(I-P) A^{-}(I-P)$, this requires $(I-P) A^{+}=$ $(I-P)\left(A^{-}-B R^{-1} B^{\prime} \Delta\right)(I-P)$. Now, $A^{-}-B R^{-1}$ $B^{\prime} \Delta=A^{+}$. Thus the equation becomes $(I-P) A^{+}=$ $(I-P) A^{+}(I-P)$. We conclude that if $A^{+P}=P A^{+} P$ and $P A^{-}=P A^{-} P$, then $\boldsymbol{\Delta}^{+} K=\Delta(I-P)$ defines a solution. Since we are only interested in symmetric solution, we also require $\boldsymbol{\Delta} \boldsymbol{P}=\boldsymbol{P}^{\prime} \boldsymbol{\Delta}$, and consequently every decomposition given in the theorem defines a solution by Lemma 8 .

Remark 16: If a matrix is diagonalizable, then a subspace is invariant if and only if it is spanned by a set of eigenvectors. Thus computation of all the solutions to the ARE involves finding $K^{+}, \Delta$, and the eigenvectors of $\boldsymbol{A}^{+}$. This procedure may be generalized to nondiagonalizable matrices using the generalized eigenvectors.

Remark 17: There are several other properties which may be derived from Lemma 7 and Theorem 6. For instance, it follows that if $K_{1}$ is a solution which yields 
$\left.A-B R^{-1}\left(B^{\prime} K_{1}+C\right)\right)$, then there exists a solution $K_{2}$, such that

$$
\begin{aligned}
\Delta\left(A-B R^{-1}\left(B^{\prime} K_{2}+C\right)\right) & = \\
& -\left(A-B R^{-1}\left(B^{\prime} K_{1}+C\right)\right) \Delta .
\end{aligned}
$$

Every solution is such that the spectrum of $A-B R^{-1}$ $\left.\left(B^{\prime} \boldsymbol{K}+\boldsymbol{C}\right)\right)$ satisfies the invariance relation

$$
\begin{aligned}
\left\{\lambda\left(A-B R^{-1}\left(B^{\prime} K+C\right)\right)\right\} & \\
\cup & \left\{-\lambda\left(A-B R^{-1}\left(B^{\prime} K+C\right)\right)\right\}=\text { constant. }
\end{aligned}
$$

In particular, $A^{+}$and $A^{-}$precisely pick out the unique solutions with $\operatorname{Re} \lambda\left(A^{+}\right)=-\operatorname{Re} \lambda\left(A^{-}\right) \leq 0$.

Remark 18: Note that when $\boldsymbol{\Delta}>\mathbf{0}$, then the solutions $\boldsymbol{K}^{+}$and $\boldsymbol{K}^{-}$are separated from the others in the sense that if $K \neq K^{+}, K^{-}$, then $\left\|K^{+}-K\right\|,\left\|K^{-}-K\right\|=$ $\|\mathbf{\Delta}\|$. The intermediate solutions may, however, be continuous deformations of one another. Such a continuum of solutions occurs if and only if $\boldsymbol{A}^{+}$has its characteristic polynomial nonequal to its minimal polynomial. Otherwise there are at most $2^{n}$ solutions (there may actually be less due to complex or repeated eigenvalues of $A^{+}$). Note that there will be a finite number of solutions if and only if $A^{+}$has a circulant vector, i.e., if there exists a vector $x$ such that $\left\{x, A^{+} x, \cdots,\left(A^{+}\right)^{n-1} x\right\}$ forms a linearly independent set. This occurs, interestingly enough, in all controllable single-input systems since the controllability of $\dot{x}=A x+b u$ implies the controllability of $\dot{x}^{*}=A^{+} x^{*}+b u$. (This corresponds to state feedback and hence preserves controllability.)

Remark 19: When $\Delta$ is merely positive semidefinite, then Theorem 6 becomes somewhat more complicated. It may be shown that

$$
\mathscr{L}^{0}\left(A-B R^{-1}\left(B^{\prime} K+C\right)\right)=\Re(\Delta)
$$

( $\mathscr{R}$ denotes null space) and is consequently independent of $\boldsymbol{K}$. Thus any solution $\boldsymbol{K}$ agrees with $\boldsymbol{K}^{+}$and $\boldsymbol{K}^{-}$on $\Re(\Delta)$. If one assumes (without loss of generality) the matrix $A^{+}$brought in the form

$$
A=\left[\begin{array}{cc}
A_{1}+ & 0 \\
0 & A_{2}{ }^{+}
\end{array}\right]
$$

with $\operatorname{Re} \lambda\left(A_{1}{ }^{+}\right)<0$ and $\operatorname{Re} \lambda\left(A_{2}{ }^{+}\right)=0$, then one may concentrate on solving the equation

$$
A_{1}{ }^{+}{ }_{\Delta_{1}}+K+\Delta_{1}+K A_{1}+=-\Delta_{1}+K B_{1} R^{-1} B_{1}{ }^{\prime} \Delta_{1}+K
$$

and this may be done using the methods of Theorem 6 . The solutions $\Delta+K$ may be written in terms of $\Delta_{1}+K$ as

$$
\left[\begin{array}{cc}
\Delta_{1}+K & 0 \\
0 & 0
\end{array}\right]
$$

The general solutions $\Delta^{+} \boldsymbol{K}$ still are of the form $\Delta(I-P)$ with $\boldsymbol{P}$ the projection satisfying the equations $\boldsymbol{P}^{\prime} \boldsymbol{\Delta}=$ $\Delta P, P A^{-} P=P A^{-}$, and $\Delta A^{+} P=\Delta P A^{+} P$. These equations are equivalent to the $A R E$, and one may work from these equations in order to determine $P$. We remark that $\Delta$ still satisfies $\Delta A^{+}+A^{-1} \Delta=0$, but that $A^{+}$and $A^{-1}$ may or may not be similar.
Remark 20: Theorem 6 is of theoretical interest in the sense that this identification of all solutions enhances the understanding of, for example, the behavior of $\mathrm{nu}$ merical algorithms for solving the ARE: However, there are certain areas of application where knowledge of all solutions of the ARE is of intrinsic importance. They lead, for instance, to network synthesis realizations which employ the minimum possible number of resistors and, in the inverse problem of covariance generation, to coloring filters with the minimum possible number of inputs.

\section{Example 2}

Consider the ARE $K^{2}=I, K=K^{\prime}$. The solutions $K^{+}$ and $K^{-}$are $I$ and $-I$, respectively. By Theorem 6 , the general solution is of the form $K=-I+2 P$, with $P$ an arbitrary orthogonal projection. Considering now the case $n=2$, we see (geometrically) that an arbitrary orthogonal projection (other than $\boldsymbol{P}=\mathbf{0}$ and $\boldsymbol{P}=\boldsymbol{I}$ which yields $K^{+}$and $K^{-}$) may be expressed as

$$
P=\left[\begin{array}{cc}
\cos ^{2} \theta & \sin \theta \cos \theta \\
\cos \theta \sin \theta & \sin ^{2} \theta
\end{array}\right]
$$

which yields the solutions

$$
K= \pm I,\left[\begin{array}{rr}
\cos 2 \theta & \sin 2 \theta \\
\sin 2 \theta & -\cos 2 \theta
\end{array}\right]
$$

with $\theta$ an arbitrary parameter. This could of course have been obtained directly from the equation $K^{2}=I$.

\section{Least Squares Stationary Optimal Control}

In this section we return to the optimal control problems discussed in Sections III and IV. With Theorem 5 and Lemma 6 at hand, the solution of the zero terminal state problem becomes straightforward.

\section{Theorem 7}

Assume that the system $\dot{x}=A x+B u$ is controllable and that $R=R^{\prime}>0$. Let $V^{+}$and $V^{-}$be as defined in Section III. Then $V^{+}>-\infty$ and $V^{-}<+\infty$ if and only if there exists a real symmetric solution to the ARE. Moreover, $V^{+}=\left\langle x, K^{+} x\right\rangle$ and $V^{-}=\left\langle x, K^{-x}\right\rangle$. The infimum in $V^{+}\left(x_{0}\right)\left(V^{-}\left(x_{0}\right)\right)$ is attained if and only if $x_{0} \in \mathscr{L}^{+}\left(A^{+}\right)\left(\mathfrak{E}^{-}\left(A^{-}\right)\right)$, and thus a minimum exists for all $x_{0}$ if and only if $K^{+}>K^{-}$. Finally, this minimum is uniquely attained by the feedback control law

$$
u^{*}=-R^{-1}\left(B^{\prime} K^{+}+C\right) x\left(-R^{-1}\left(B^{\prime} K^{-}+C\right) x\right)
$$

and leads to the closed-loop response equations

$$
\begin{aligned}
\dot{x}^{*} & =A^{+} x^{*}\left(\dot{x}^{*}=A^{-} x^{*}\right) \\
x^{*}(0) & =x_{0} .
\end{aligned}
$$

Proof: Assume first that $K^{+}>K^{-}$. Then $\operatorname{Re} \lambda\left(A^{+}\right)<0$. By Lemma 6,

$$
\begin{aligned}
\eta=\left\langle x_{0}, K^{+} x_{0}\right\rangle+\int_{0}^{\infty}\left\|u+R^{-1}\left(B^{\prime} K+C\right) x\right\|_{R^{2}} d t \\
\geq\left\langle x_{0}, K^{+} x_{0}\right\rangle
\end{aligned}
$$


for all $u \in L_{2 e^{+}}$such that $\lim _{t \rightarrow \infty} x(t)=0$. Thus $V^{+}=$ $\left\langle x_{0}, K^{+} x_{0}\right\rangle$ and equality is attained if and only if $u=$ $-R^{-1}\left(B^{\prime} K+C\right) x$ almost everywhere.

Assume now that $\boldsymbol{K}^{+} \geq \boldsymbol{K}^{-}$. Substituting $Q+\epsilon \boldsymbol{I}$, $\epsilon>0$, for $Q$ yields $K_{\epsilon}{ }^{+}>K_{\epsilon}^{-}$. Letting $\epsilon \rightarrow 0$ yields $\lim _{\epsilon \downarrow 0}$ $K_{\epsilon}^{+} \downarrow K^{+}$and $\lim _{\epsilon \downarrow 0} K_{\epsilon}^{--} \uparrow K^{-}$, and the result in this case follows by continuity of $\eta(\epsilon)$ for fixed $u$. The theorem is proven in an analogous way for $V^{-}$.

As is shown in the foregoing, the fixed endpoint case causes no difficulties. The free endpoint problem is more subtle since, even if the infimum exists, it may very well be that the closed-loop system is unstable. The following theorem shows that this will only happen exceptionally.

\section{Theorem 8}

Assume that the system $\dot{x}=A x+B u$ is controllable and that $R=R^{\prime}>0$. Let $V_{f}{ }^{+}$be as defined in Section III. Then $V_{f}{ }^{+}>-\infty$ if and only if there exists a real symmetric nonpositive definite solution to the ARE. Thus $V_{f^{+}}{ }^{+}>-\infty$ if and only if $K^{-} \leq \mathbf{0}$. Noreover, when $\boldsymbol{K}^{-}<\mathbf{0}$, then $V_{f}^{+}=V^{+}=\left\langle x, \boldsymbol{K}^{+} \boldsymbol{x}\right\rangle$. Finally, this infimum is a minimum, and the optimal closed-loop system $\dot{x}^{*}=$ $\boldsymbol{A}^{+} \boldsymbol{x}^{*}$ is asymptotically stable if the combined inequalities $K^{-}<0$ and $K^{+}>K^{-}$hold.

Proof: By Theorem 1, $V_{f}{ }^{+}>-\infty$ if and only if $V^{-} \leq$ 0 . The first part of the theorem thus follows from Theorem 7. Assume now that $\boldsymbol{K}^{-}<\mathbf{0}$ and $\boldsymbol{K}^{+}>\boldsymbol{K}^{-}$. We then need to show that $\lim _{t \rightarrow-\infty} K(t)=K^{+}$, with $K(t)$ the solution to the Riccati differential equation

$$
\dot{K}=-A^{\prime} K-K A+\left(K B+C^{\prime}\right) R^{-1}\left(B^{\prime} K+C\right)-Q
$$

with initial condition $K(0)=0$. Let $\Delta^{-} K=K-K^{-}$. Then $\boldsymbol{\Delta}-\boldsymbol{K}$ satisfies

$$
\frac{d}{d t} \mathbf{\Delta}^{-} \boldsymbol{K}=-A^{-1} \mathbf{\Delta}^{-} \boldsymbol{K}-\mathbf{\Delta}^{-} \boldsymbol{K} A^{-}+\Delta^{-} K B R^{-1} B^{\prime} \Delta^{-K}
$$

and $\Delta^{-} K(0)=-K^{-}>0$. This differential equation has an obvious variational interpretation in terms of a finite time optimal control problem with a penalty on the terminal state. This shows that $\Delta-K(t)$ exists and is positive definite on $t \leq 0$. Thus $\mathbf{\Sigma}=\left(\Delta^{-} K\right)^{-1}$ exists for $t \leq 0$ and satisfies $\dot{\mathbf{\Sigma}}=A^{-\mathbf{\Sigma}}+\mathbf{\Sigma} A^{-1}-B R^{-1} B^{\prime}$. Let $\mathbf{\Sigma}_{\infty}=\left(K^{+}-K^{-}\right)^{-1}$. Thus $A^{-} \mathbf{\Sigma}_{\infty}+\mathbf{\Sigma}_{\infty} A^{-1}=B R^{-1} B^{\prime}$, and $\boldsymbol{\Delta} \mathbf{\Sigma}=\mathbf{\Sigma}-\mathbf{\Sigma}_{\infty}$ satisfies $(d / d t) \mathbf{\Delta} \mathbf{\Sigma}=\boldsymbol{A}^{-\boldsymbol{\Delta}} \mathbf{\Sigma}+$ $\boldsymbol{\Delta} \mathbf{\Sigma} A^{-1}$. Since $\operatorname{Re} \lambda\left(\boldsymbol{A}^{-}\right)>0$, this shows that $\lim _{t \rightarrow-\infty}$ $\Delta \mathbf{\Sigma}(t)=\mathbf{0}$ and hence $\lim _{t \rightarrow-\infty} \boldsymbol{K}(t)=\boldsymbol{K}^{+}$. Consequently $V_{f}^{+}=V^{+}=\left\langle x, K^{+} x\right\rangle$ when $K^{-}<\mathbf{0}$ and $\boldsymbol{K}^{+}>\boldsymbol{K}^{-}$. It remains to be shown that this also holds when $K^{+} \geq K^{-}$ and $K^{-}<0$. This again follows from a continuity argument by letting $\epsilon \downarrow 0$ after substituting $Q+\epsilon I$ for $Q$.

Remark 21: Examination of the proof of Theorem 8 shows that the Riccati differential equation

$$
\dot{K}=-A^{\prime} K-K A+\left(K B+C^{\prime}\right) R^{-1}\left(B^{\prime} K+C\right)-Q
$$

yields $\lim _{t \rightarrow-\infty} K(t)=K^{+}$, when $K(0)>K^{-}$and $\lim _{t \rightarrow \infty}$ $\boldsymbol{K}(t)=\boldsymbol{K}^{-}$when $\boldsymbol{K}(0)<\boldsymbol{K}^{+}$. (In order to show the

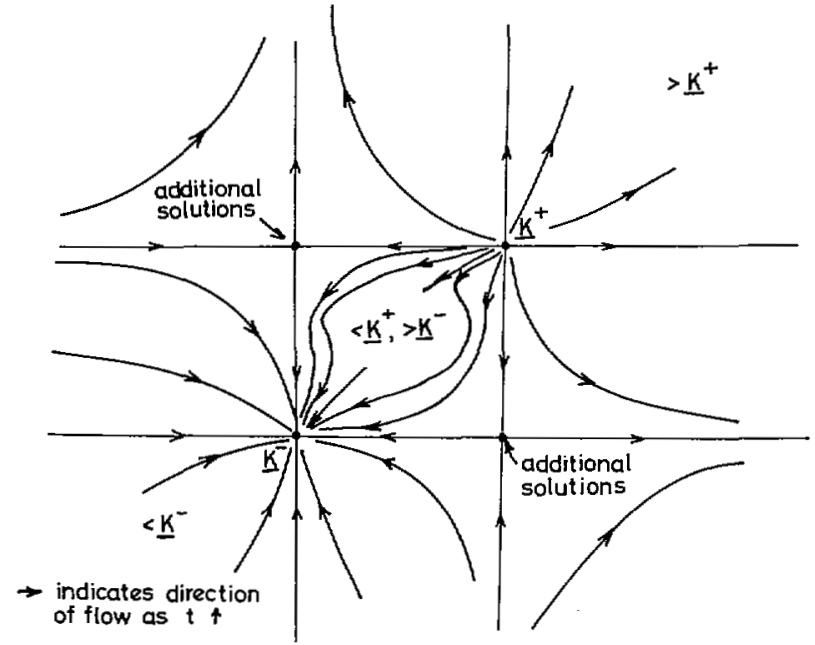

Fig. 2. Behavior of Riccati differential equation.

convergence required in Theorem 8 we needed to make sure that $\boldsymbol{K}(0)=\mathbf{0}>\boldsymbol{K}^{-}$.) A more detailed analysis shows that the Riccati differential equation behaves qualitatively as is schematically shown in Fig. 2. (This figure is to be interpreted with imagination since two-dimensional pictures never represent flows in the space of nonscalar symmetric matrices.) Of particular interest are the local stability conditions of the equilibria. It is casy to see that if $\operatorname{Re} \lambda\left(A^{+}\right)<0$, then $K^{+}$is locally asymptotically stable in the negative time direction, and $\boldsymbol{K}^{-}$is locally asymptotically stable in the forward time direction. None of the other solutions enjoy these stability properties. Thus if the Riccati differential equation approaches a limit $\lim _{t \rightarrow-\infty}$ $K(t) \neq K^{+}$, then the backward integration is unstable at this limit.

Remark 22: The Newton-Raphson algorithm provides a suitable method for solving the ARE [17], [18]. It may thus be shown that an initial choice $K_{0}=K_{0}^{\prime}$ such that

$$
\operatorname{Re} \lambda\left(A-B R^{-1}\left(B^{\prime} K_{0}+C\right)\right)<0
$$

yields a well-defined series

$$
\begin{aligned}
\boldsymbol{K}_{1}=\boldsymbol{K}_{1}{ }^{\prime}>\boldsymbol{K}_{2} & =\boldsymbol{K}_{2}{ }^{\prime}>\cdots>\boldsymbol{K}_{n} \\
& =\boldsymbol{K}_{n}{ }^{\prime}>\cdots>\boldsymbol{K}^{+}, \quad \text { with } \lim _{n \rightarrow \infty} \boldsymbol{K}_{n}=\boldsymbol{K}^{+}
\end{aligned}
$$

and that an initial choice with

$$
\operatorname{Re} \lambda\left(A-B R^{-1}\left(B^{\prime} K_{0}+C\right)\right)>0
$$

yields a well-defined series

$$
\begin{array}{r}
\boldsymbol{K}_{1}=K_{1}{ }^{\prime}<\boldsymbol{K}_{2}=\boldsymbol{K}_{2}{ }^{\prime}<\cdots<K_{n}=K_{n}{ }^{\prime}<\cdots<K^{-} \\
\text {with } \lim _{n \rightarrow \infty} K_{n}=K^{-} .
\end{array}
$$

Remark 23: A good example of the pathological cases which Theorem 8 does not deal with is given by the trivial optimization problem which consists in minimizing $\int_{0}^{\infty}\|u\|^{2} d t$. The relevant Riccati equation becomes

$$
-A^{\prime} K-K A+K B B^{\prime} K=0 .
$$

Thus $\boldsymbol{K}=\mathbf{0}$ is a solution; it is in fact the solution which leads to the free terminal time optimal control. It is the maximum (minimum) solution if $\operatorname{Re} \lambda(A) \leq 0(\geq 0)$. 
Otherwise $\boldsymbol{K}=\mathbf{0}$ will be an intermediate solution and the Riccati differential equation fails to converge to $K^{+}$. When $\operatorname{Re} \lambda(A) \neq 0$ and one eigenvalue has positive real part, then the closed-loop response is not stable even though there exists a solution to the ARE which stabilizes the closed-loop system.

Remark 24: Let

$$
\eta^{*}=\lim _{T \rightarrow \infty} \inf _{u \in L_{2 e} e^{+}} \int_{0}^{T} w(x, u) d t
$$

subject to the constraints $\dot{x}=A x+B u, x(0)=x_{0}$, and $x(T)=x_{\infty}$. It mayy be shown that $\eta^{*}>-\infty$ if the conditions of Theorem 7 are satisfied. In fact,

$$
\eta^{*}=\left\langle x_{0}, K^{+} x_{0}\right\rangle-\left\langle x_{\infty}, K-x_{\infty}\right\rangle
$$

This shows that the condition $K^{-} \leq \mathbf{0}$ is a necessary condition for the free endpoint problem to have a solution. Assuming now that $K^{-} \leq 0$, then we may be lead to think that by minimizing $\eta^{*}$ over $x_{\infty}$, one obtains the infimum of the free endpoint control problem. This is the case if $K^{-}<0$ but not necessarily otherwise, as shown by the example in the previous remark.

Remark 25: The condition $K^{-}<0$ as a condition for the solution of the free endpoint problem is a considerable improvement over [3, theorem 23.6]. This reference gives the condition $K^{+}<0$ as a sufficient condition for the existence of a minimum. Since $\boldsymbol{K}^{-} \leq \boldsymbol{K}^{+}$, this condition is thus a special case of ours.

Remark 26: The ARE loses its significance in singular control problems, i.e., when $R$ is a singular matrix. A very convenient way of treating such problems is by replacing $R$ by $R+\epsilon I, \epsilon>0$, and letting $\epsilon \downarrow 0$. If the infimum is bounded at $\epsilon=0$, then it is bounded for $\epsilon \geq 0$ and thus $\boldsymbol{K}_{\epsilon}{ }^{+}$and $\boldsymbol{K}_{\epsilon}-$ are well defined for $\epsilon>0$ as the maximum and minimum solution to the ARE, with $R$ replaced by $R+\epsilon I$. Since $K_{c}^{+}$and $K_{t}^{-}$are monotone functions of $\epsilon$, it follows that $\lim _{\epsilon \downarrow \infty} K_{\epsilon}^{+}$and $\lim _{\epsilon \downarrow \infty \infty} K_{\epsilon}^{-}$ exist. Of course these limits are not solutions of a Riccati equation but are the maximum and the minimum solution to the LMI. Thus by defining $K^{+}$and $K^{-}$in this way, one may conclude that $V^{+}=\left\langle x, K^{+} x\right\rangle$ and

$$
V^{-}=\left\langle x, K^{-} x\right\rangle
$$

also in the singular case.

\section{Conclusions}

Let us briefly recapitulate the major results of this paper. We use the notation introduced in Sections II and III.

Consider first the infinite-time least squares optimal control problem with the stability condition $\lim _{t \rightarrow \infty}$ $x(t)=0$ as an explicit constraint. Then the condition $V^{+}>-\infty$ (i.e., boundedness of the infimum) is equivalent to any of the following conditions: $\mathscr{S} w(x, u) d t \geq$ 0 , i.e., this inequality holds for all $u$ such that $x(0)=$ $x(T) ; V^{-}<+\infty$; there exists as $V$ a function which satisfies the DIE; the FDI $H(-j \omega, j \omega) \geq 0$ is valid for all real $\omega$; there exists a real symmetric solution to the
LMI; there exists a real symmetric solution to the QMI; and there exists a real symmetric solution to the ARE. (The last two statements make sense only if $\boldsymbol{R}>\mathbf{0}$.)

The value of $V+$ is given by the quadratic form $V^{+}=$ $\left\langle x, \boldsymbol{K}^{+} \boldsymbol{x}\right\rangle$, where $\boldsymbol{K}^{+}$is the maximum real symmetric solution of either the LMI, the QMI, or the ARE. This infimum may not be a minimum because the optimal closed-loop system

$$
\dot{x}^{*}=\left(A-B R^{-1}\left(B^{\prime} K+C\right)\right) x^{*}
$$

may not be asymptotically stable. It is asymptotically stable if and only if $\boldsymbol{K}^{+}>\boldsymbol{K}^{-}$, where $\boldsymbol{K}^{-}$is the minimum real symmetric solution of either the LMII, the QMI, or the ARE. In frequency domain terms this condition requires that there exists an $\epsilon>0$ such that

$$
H(-j \omega, j \omega) \geq \epsilon B^{\prime}\left(-I j \omega-A^{\prime}\right)^{-1}(I j \omega-A)^{-1} B
$$

for all real $\omega$.

Consider now the infinite time least squares optimal control problems without restrictions on the behavior of $x(t)$ at $\infty$. Then the condition $V_{f}^{+}>-\infty$ (i.e., the boundedness of the infimum) is equivalent to any of the following conditions: $\int_{0}^{T} w(x, u) d t \geq 0$, whenever $x(0)=$ $0 ; V^{-} \leq 0 ; V_{n}{ }^{+}>-\infty$; there exists a $V$ function $V \leq 0$ which satisfies the DIE; $H(\bar{s}, s) \geq 0$ throughout Re $s \geq 0$; there exists a real symmetric nonpositive definite solution to the LIII; or to the QMI; or to the ARE. A general characterization of the value of $V_{f}+$ is not known. When $\boldsymbol{K}^{-}<\mathbf{0}$, then $V_{t}^{+}=V^{\div}$. In frequency-domain terms this requires that there exists an $\epsilon>0$ such that

$$
H(\bar{s}, s) \geq \epsilon(s+\bar{s}) B^{\prime}\left(\bar{I}-A^{\prime}\right)^{-1}(I s-A)^{-1} B
$$

for $\operatorname{Re} s \geq 0$. The infimum $V_{f}{ }^{+}=V^{+}$is attained, and the optimal closed-loop system

$$
\dot{x}^{*}=\left(A-B R^{-1}\left(B^{\prime} K+C\right)\right) x^{*}
$$

is asymptotically stable if the strict inequalities $K^{-}<0$ and $\boldsymbol{K}^{+}>\boldsymbol{K}^{-}$hold.

We conclude with two remarks which contrast the results with the ideas based on spectrum factorization and the methods based on the Hamiltonian matrix.

Remark 27: Consider the following equation in the rational matrix $W(s)$ :

$$
H(-s, s)=W^{\prime}(-s) W(s) .
$$

This factorization equation (FE) plays a very important role in applied mathematics. Its relevance in filtering and prediction is well known through the work of Wiener [19], and it has been developed as a method for solving infinite time least squares optimal control problems by Brockett [20]. The theoretical aspects of the FE have been studied in great generality by Youla [21]. For a state-space interpretation of these results, see the work of Anderson (e.g., [22]).

It is usually assumed that the $F E$ is, in some sense, the analog of the ARE. This is true up to a point but, as we shall see, the relationship with the LMII goes a lot further.

Consider first the LMII and assume that $K=K^{\prime}$ is a solution. Let $F(K)$ be factored as 


$$
\left[\begin{array}{cc}
A^{\prime} K+K A+Q & K B+C^{\prime} \\
B^{\prime} K+C & R
\end{array}\right]=\left[\begin{array}{l}
M^{\prime} \\
N^{\prime}
\end{array}\right]\left[\begin{array}{ll}
M & \cdot
\end{array}\right]
$$

i.e., $d(x, u)=\|M x+N u\|^{2}$ is the "dissipation rate" since it satisfies

$$
\frac{d}{d t}\langle x, K x\rangle+w(x, u)=d(x, u)
$$

along solutions of $\dot{x}=A x+B u$. Premultiplying this equation by $\left[\begin{array}{ll}B^{\prime}\left(-I s-A^{\prime}\right)^{-1} & I\end{array}\right]$ and postmultiplying by

$$
\left[\begin{array}{c}
(I s-A)^{-1} B \\
I
\end{array}\right]
$$

shows that

$$
W(s)=N+M(I s-A)^{-1} B
$$

satisfies the FE. The converse is true as well, i.e., if $N+$ $\boldsymbol{M}(\boldsymbol{I} s-\boldsymbol{A})^{-1} \boldsymbol{B}$ satisfies the FE, then

$$
F(K)=\left[\begin{array}{l}
M^{\prime} \\
N^{\prime}
\end{array}\right][M N] .
$$

To show the special role played by the solutions of the ARE, assume that $R>\mathbf{0}$ and let $q$ be the number of rows of $M$ and $N$. Then $q \geq \operatorname{rank} R$. In fact, a simple calculation shows that we may take $q=\operatorname{rank} R$ if and only if $K$ is a solution to the ARE. The matrix $M$ is then given in terms of $K$ and $N$ by $M=N R^{-1}\left(B^{\prime} K+C\right)$. Thus

$$
W(s)=N\left(I+R^{-1}\left(B^{\prime} K+C\right)(I s-A)^{-1} B\right)
$$

with $N$ an $(m \times m)$ matrix which satisfies $N^{\prime} N=R$ and $K$ a solution to the ARE, defines the set of solutions of the FE for which $W(s)$ is a square $(m \times m)$ matrix, i.e., $W$ has the minimum number of rows.

Consider now the roles of $\boldsymbol{K}^{+}$and $\boldsymbol{K}^{-}$. Since

$$
\begin{aligned}
& {\left[I+R^{-1}\left(B^{\prime} K+C\right)(I s-A)^{-1} B\right]^{-1}=I } \\
&-R^{-1}\left(B^{\prime} K+C\right)\left(I s-A+B R^{-1}\left(B^{\prime} K+C\right)\right)^{-1} B
\end{aligned}
$$

it follows that $W(s)$ is square and that its inverse is analytic in $\operatorname{Re} s>0(<0)$ if it is generated by $\boldsymbol{K}^{+}\left(\boldsymbol{K}^{-}\right)$. By Youla's result [21, theorem 2] it follows that such a $W(s)$ is unique. Thus

$$
W^{+}(s)=N\left(I+R^{-1}\left(B^{\prime} K^{+}+C\right)(I s-A)^{-1} B\right)
$$

with $N$ an $(m \times m)$ matrix satisfying $N^{\prime} N=R$, yields the so-called "spectral factorization" of $H(-s, s)$, and

$$
W^{-}(s)=N\left(I+R^{-1}\left(B^{\prime} K^{-}+C\right)(I s-A)^{-1} B\right)
$$

yields the "anti-spectral factorization."

In conclusion, every solution to the LMI generates a solution to the $\mathrm{FE}$, and the solutions of the ARE are the minimum rank solutions. Note, however, that this does not yet exhaust the set of solutions to the FE since we are restricting $W(s)$ to have its singularities at the eigenvalues of $\boldsymbol{A}$. Finally, let it be mentioned that the nature of the solution $W^{+}(s)$ may be given as a very general operator theoretic interpretation when $\operatorname{Re} \lambda(A)<0$, but that it is hard to see mathematically what is going on when this is not the case.
Remark 28: Other than the LMI, the QMI, and the ARE, or the $\mathrm{FE}$, there is a third avenue of approah to the problems under consideration, namely, via the state-costate two-point boundary value problem which results from the application of Pontryagin's minimum principle. This approach leads to the matrix

$$
Z=\left[\begin{array}{cc}
A-B R^{-1} C & -B R^{-1} B^{\prime} \\
C^{\prime} R^{-1} C-Q & -A^{\prime}+C^{\prime} R^{-1} B^{\prime}
\end{array}\right]
$$

Let

$$
J=\left[\begin{array}{rr}
0 & I \\
-I & 0
\end{array}\right] .
$$

Then $J Z=(J Z)^{\prime}$, i.e., $Z$ is a Hamiltonian matrix.

Note that

$\operatorname{det}(I s-Z)=(-1)^{n} \operatorname{det}\left(-I s-A^{\prime}\right)$ $\cdot \operatorname{det} H(-s, s) \operatorname{det}(\boldsymbol{I} s-\boldsymbol{A})$.

Thus $(-1)^{n} \operatorname{det}(I s-Z)$ may be spectral factored as $\Delta(-s) \Delta(s)$. A computational algorithm for solving the ARE based on $\Delta(s)$ is outlined in [18]. This algorithm leads to $K^{+}$, when $\Delta(s)$ is taken to be Hurwitz, and to $K^{-}$, when $\Delta(-s)$ is taken to be Hurwitz.

Another method of solving the ARE which is based on the Hamiltonian matrix $Z$ has been introduced in [23] and more generally by Potter in [24]. Potter's analysis was based on the restriction that $Z$ has distinct eigenvalues. This assumption may be removed as shown in [14]. This technique is based on the observation that the ARE may be written as

$$
\left[\begin{array}{ll}
I & K
\end{array}\right] J Z\left[\begin{array}{l}
I \\
K
\end{array}\right]=0 .
$$

From this it is easy to see that $K$ is a solution of the ARE if and only if there exists an $(n \times n)$ matrix $L$ such that

$$
Z\left[\begin{array}{l}
I \\
K
\end{array}\right]=\left[\begin{array}{l}
I \\
K
\end{array}\right] L
$$

i.e., the vectors in the matrix

$$
\left[\begin{array}{l}
I \\
K
\end{array}\right]
$$

span an invariant subspace of $Z$. Thus if the vectors in

$$
\left[\begin{array}{l}
M \\
N
\end{array}\right]
$$

span an invariant subspace of $Z$ and $M^{-1}$ exists, then $N M^{-1}$ is a solution. Symmetry of $K$ implies that this subspace $S$ satisfies the additional condition $S^{\perp}=J S$. Based on these properties one may then construct all the solutions of the ARE. In terms of the eigenvectors

$$
\boldsymbol{e}_{i}=\left[\begin{array}{l}
f_{i} \\
g_{i}
\end{array}\right] \text { of } Z
$$

this yields

$$
K=\left[\begin{array}{llll}
g_{1} & g_{2} & \cdots & g_{n}
\end{array}\right]\left[\begin{array}{llll}
f_{1} & f_{2} & \cdots & f_{n}
\end{array}\right]^{-1}
$$


(assuming this inverse exists) as the general solution of the ARE. The corresponding eigenvalues of $Z$ are also the eigenvalues of the closed-loop system matrix $A-B R^{-1}$ $\left(B^{\prime} K+C\right)$, and the vectors $g_{1}, g_{2}, \cdots, g_{n}$ are the corresponding eigenvectors. The matrix $K$ is Hermitian if $\lambda_{i}+\bar{\lambda}_{j} \neq 0$, and real if $\lambda_{1}$ and $\bar{\lambda}_{i}$ are simultaneously included in these eigenvalues. This method produces the matrix $\boldsymbol{K}^{+}$if $\operatorname{Re} \lambda_{i}<0$ and $\boldsymbol{K}^{-}$if $\operatorname{Re} \lambda_{i}>0$. For complete details, see [14].

The classification of all the solutions to the ARE provided by this method is similar to that discussed in Section VI. It is in fact quite easy to spell out the parallel in both methods. Although the method based on the Hamiltonian matrix provides an important procedure for numerical computation of $\boldsymbol{K}^{+}$, we feel that as a means of classifying all the solutions of the ARE, it fails to provide the insight and lacks the explicitness which the results of Section VI offer. The computational methods which have been proposed based on the preceding characterization of $\mathrm{K}^{+}$require computing the eigenvalues and eigenvectors of $\mathrm{Z}$. Note, however, that this procedure generates a lot of unnecessary information since what one is really interested in is determining the invariant subspace of $Z$ associated with its left half-plane eigenvalues. Roberts [25] has recently developed an elegant technique which achieves precisely this.

\section{REFERENCES}

[1] R. E. Kalman, "Contributions to the theory of optimal control," Bol. Soc. Mat. Mex., vol. 5, pp. 102-199, 1960.

[2] G. C. Newton, Jr., L. A. Gould, and J. F. Kaiser, Analytic Design of Linear Feedback Controls. New York: Wiley, 1957.

[3] R. W. Brockett, Finite Dimensional Linear Systems. New York: Wiley, 1970.

[4] R. E. Kalman, "When is a linear system optimal?" Trans. ASME, J. Basic Eng., ser. D., vol. 86, pp. 51-60, 1964.

[5] - "Lyapunov functions for the problem of lurie in automatic control," in Proc. Nat. Acad. Sci. U.S.A., vol. 49, pp. 201-205, 1963.

[6] V. M. Popov, "Hyperstability and optimality of automatic systems with several control functions," Rev. Roum. Sci. Tech., Ser. Electrotech. Energ., vol. 9, pp. 629-690, 1964.

[7] B. D. O. Anderson, "A system theory criterion for positive real matrices," SIA $M J$. Contr., vol. 5, pp. 171-182, 1967.

[8] B. D. O. Anderson and J. B. Moore, "Algebraic structure of generalized positive real matrices," SIAM J. Contr., vol. 6, pp. $615-623,1968$.

[9] J. C. Willems, "Dissipative dynamical systems," to be published.

[10] R. W. Brockett, and H. B. Lee, "Frequency-domain instability criteria for time-varying and nonlinear systems," Proc. IEEE, vol, 55, pp. 604-619, May 1967.

[11] D. L. Kleinman, "On the linear regulator problem and the matrix Riccati equation," Electron. Syst. Lab., M.I.T., Cambridge, Rep. ESL-R-271, 1966.

[12] R. S. Bucy, "Global theory of the Riccati equation," J. Comput. Syst. Sci., vol. 1, pp. 349-361, 1967.

[13] W. M. Wonham, "On a matrix Riccati equation of stochastic control," SIAM J. Contr., vol. 14, pp. 681-698, 1968.

[14] K. Mârtensson, "On the matrix Riccati equation," Inform. Sci., vol. 3, pp. 17-49, 1971.

[15] J. C. Willems, "The generation of Lyapunov functions for input-output stable systems," SIAM J. Contr., vol. 9, pp. $105-134,1971$.

[16] R. W. Brockett, "Structural properties of the equilibrium solutions of Riccati equations," Springer-Verlag Lecture Notes on Wath., vol. 132, pp. 61-69, 1970.

[17] D. L. Kleinman, "On an iterative technique for Riccati equation computations," IEEE Trans. Automat. Contr. (Corresp.), vol. AC-13, pp. 114-115, Feb. 1968.

[18] R. S. Bucy and P. D. Joseph, Filtering for Stochastic Processes with Applications to Guidance. New York: Interscience, 1968.

[19] N. Wiener, Extrapolation, Interpolation and Smoothing of Statimary Time Series with Engineering Applications. New York: Wiley, 1949.

[20] R. W. Brockett, "Path integrals, Lyapunov functions, and quadratic minimization," in Proc. Lth Annu. Allerton Conf. Circuit and System Theory, pp. 685-698, 1966.

[21] D. C. Youla, "On the factorization of rational matrices," IRE Trans. Inform. Theory, vol. IT-7, pp. 172-189, July 1961.

[22] B. D. O. Anderson, "The inverse problem of stationary covariance generation," J. Statist. Phys., vol. 1, pp. 133-147, 1969.

[23] A. G. J. MacFarlane, "An eigenvector solution of the optimal linear regulator problem," $J$. Ellectron. Contr., vol. 14, pp. 496-501, 1963

[24] J. E. Potter, "Matrix quadratic solutions," SIAM J. Appl. Math., vol. 14, pp. 496-501, 1966.

[25] J. D. Roberts, "Linear model reduction and solution of the algebraic Riccati equation by use of the sign function," Dep. Eng., Univ. Cambridge, Cambridge, England, Rep. CUED/BControl/TR 13, 1971 .

[26] D. H. Jacobson, "A new necessary condition of optimality for singular control problems," SIAM $J$. Contr., vol. 7, pp. 578$59 \overline{5}, 1969$.

Jan C. Willems (S'66-M'68), for a photograph and biography please see this issue, page 595 . 